\title{
Bis(heterocumulenes) Derived from the 1,4-Diphenyl-1,3- butadiyne Framework. Synthesis of Three New Classes of Axially Chiral Biheteroaryls
}

\author{
Mateo Alajarín, ${ }^{* a}$ Baltasar Bonillo, ${ }^{\text {a Ángel Vidal,*a }}$ \\ Delia Bautistab \\ ${ }^{a}$ Departamento de Química Orgánica, Facultad de Química, Universidad de Murcia, \\ Campus de Espinardo, 30100, Murcia, Spain \\ ${ }^{b}$ Servicio Universitario de Instrumentación Científica, Universidad de Murcia, Campus de \\ Espinardo, 30100, Murcia, Spain
}

Table of Contents

General Methods and Materials....................................... S2

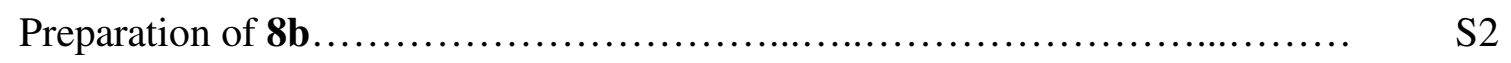

Preparation of Bis(iminophosphoranes) 9........................... S2-S3

Analytical and Spectroscopic Data of Bis(benzocarbazole) 10b .............. S3

Analytical and Spectroscopid Data of Bis(quinindolines) 11b-d.............. S4

Preparation of Iminophosphorane 12................................ S4-S5

Preparation of Benzocarbazole-Quinindolines 13....................... S5-S6

Analytical and Spectroscopic Data of Benzocarbazole-Quinindoline 14b....... S6

Figure S1. X-Ray structure of 11 a................................... S7

Figure S2. X-Ray structure of 13a................................... S7

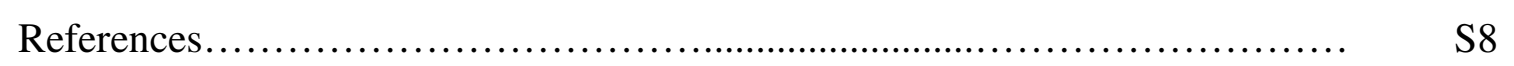

Copy of ${ }^{1} \mathrm{H}-\mathrm{NMR}$ spectra of compounds 10, 11, 13 and 14...... S9-S18

Copy of ${ }^{1} \mathrm{H}-\mathrm{NMR}$ spectrum of 11a $+(S)$-TFAE (4 equiv) S19-S21

Copy of ${ }^{13} \mathrm{C}$-NMR spectra of compounds 10, 11, 13 and $14 \ldots \ldots . . \quad$ S22-S31 


\section{General Methods}

All melting points are uncorrected. Infrared (IR) spectra were recorded neat or as Nujol emulsions. ${ }^{1} \mathrm{H}$ NMR spectra were recorded in $\mathrm{CDCl}_{3}$ at 300 or $400 \mathrm{MHz} .{ }^{13} \mathrm{C}$ NMR spectra were recorded in $\mathrm{CDCl}_{3}$ at 75 or $100 \mathrm{MHz}$. The chemical shifts are expressed in ppm, relative to $\mathrm{Me}_{4} \mathrm{Si}$ at $\delta=0.00 \mathrm{ppm}$ for ${ }^{1} \mathrm{H}$, while the chemical shifts for ${ }^{13} \mathrm{C}$ are reported relative to the resonance of $\mathrm{CDCl}_{3} \delta=77.10 \mathrm{ppm}$ or DMSO- $d_{6} \delta=35.35 \mathrm{ppm}$.

Materials: $\quad$ 2-Ethynyl-4-methylaniline $\quad \mathbf{7 b},{ }^{1} \quad$ 2-[4-(2-aminophenyl)-1,3butadiynyl]phenylamine $\mathbf{8 a},{ }^{2}$ and diphenylketene ${ }^{3}$ were prepared following published experimental procedures.

\section{Preparation of 2-[4-(2-amino-5-methylphenyl)-1,3-butadiynyl]-4-methylphenylamine 8b}

$\mathrm{Cu}(\mathrm{OAc})_{2} \cdot \mathrm{H}_{2} \mathrm{O}(3.99 \mathrm{~g}, 20 \mathrm{mmol})$ was dissolved in pyridine $(25 \mathrm{~mL})$ and methanol $(25 \mathrm{~mL})$ and 2-ethynyl-4-methylaniline $7 \mathbf{b}(1.31 \mathrm{~g}, 10 \mathrm{mmol})$ was added. The reaction mixture was stirred at room temperature for $24 \mathrm{~h}$. The solution was treated with water $(300 \mathrm{~mL})$, and extracted with diethyl ether $(3 \times 150 \mathrm{~mL})$. The combined organic layers were washed with aqueous CuSO4 solution $(350 \mathrm{~mL})$, water $(3 \times 300 \mathrm{~mL})$ and dried over anhydrous $\mathrm{MgSO}_{4}$. After removing the solvent under reduced pressure the resulting solid was triturated with diethyl ether $\left(30 \mathrm{~mL}\right.$ ), filtered and dried under vacuum. Yield 96\%; mp $193{ }^{\circ} \mathrm{C}$ (colorless prisms, diethyl ether); IR (Nujol) 3450, 3324, 2133, 1623, 1610, 1314, 1306, 1258, 1157, 1042, 1002, 941, 875, 806, $724 \mathrm{~cm}^{-1} ;{ }^{1} \mathrm{H}$ NMR $\left(\mathrm{CDCl}_{3}, 300 \mathrm{MHz}\right) \delta 2.11(\mathrm{~s}, 3 \mathrm{H}), 5.39$ (s, 2 $\mathrm{H}), 6.64(\mathrm{~d}, 1 \mathrm{H}, J=8.3 \mathrm{~Hz}), 6.92(\mathrm{dd}, 1 \mathrm{H}, J=8.3,2.0 \mathrm{~Hz}), 7.02(\mathrm{~d}, 1 \mathrm{H}, J=2.0 \mathrm{~Hz}) ;{ }^{13} \mathrm{C}$ NMR ( $\left.\mathrm{CDCl}_{3}, 75 \mathrm{MHz}\right) \delta$ 19.7, 78.6 (q), 80.7 (q), 103.7 (q), 114.4, 124.5 (q), 131.7, 132.1, 149.2 (q); MS (EI, $70 \mathrm{eV}) \mathrm{m} / z$ (rel int) $259\left(\mathrm{M}^{+}, 100\right)$. Anal. Calcd for $\mathrm{C}_{18} \mathrm{H}_{16} \mathrm{~N}_{2}$ (260.34): $\mathrm{C}$, 83.05; H, 6.19; N, 10.76. Found: C, 83.25; H, 6.08; N, 10.65.

\section{Preparation of Bis(iminophosphoranes) 9}

To a solution of the diamine $8(3 \mathrm{mmol})$ in anhydrous acetonitrile $(10 \mathrm{~mL})$ were added triphenylphosphine $(3.15 \mathrm{~g}, 12 \mathrm{mmol})$, triethylamine $(6 \mathrm{~mL})$ and carbon tetrachloride $(4 \mathrm{~mL})$. The resulting mixture was maintained at room temperature without stirring for $12 \mathrm{~h}$. The precipitated solid was filtered, washed with cold water $(3 \times 50 \mathrm{~mL})$ to remove the triethylammonium chloride, and dried under vacuum to afford the bis(iminophosphorane) 7 as crystalline solid. 
Bis(iminophosphorane) 9a $\left(\mathbf{R}^{\mathbf{1}}=\mathbf{H}\right)$ : Yield 93\%; mp $227{ }^{\circ} \mathrm{C}$ (yellow prisms, acetonitrile); IR (Nujol) 2200, 2130, 1586, 1273, 1183, 1153, 1106, 1047, 1019, 996, 933, 770, 755, 742, 716, $697 \mathrm{~cm}^{-1} ;{ }^{1} \mathrm{H} \mathrm{NMR}\left(\mathrm{CDCl}_{3}, 300 \mathrm{MHz}\right) \delta 6.46(\mathrm{~d}, 2 \mathrm{H}, J=8.1 \mathrm{~Hz}), 6.60(\mathrm{t}, 2 \mathrm{H}, J=7.4$ $\mathrm{Hz})$, 6.84-6.90 (m, $2 \mathrm{H}), 7.36-7.45(\mathrm{~m}, 20 \mathrm{H}), 7.80-7.89(\mathrm{~m}, 12 \mathrm{H}) ;{ }^{13} \mathrm{C} \mathrm{NMR}\left(\mathrm{CDCl}_{3}, 75\right.$ MHz) $\delta 78.1$ (q), $83.2(\mathrm{q}), 117.1,118.3(\mathrm{q}, \mathrm{d}, J=22.5 \mathrm{~Hz}), 121.4(\mathrm{~d}, J=9.2 \mathrm{~Hz}), 128.6(\mathrm{~d}, J=$ $12.1 \mathrm{~Hz}), 128.9,131.1(\mathrm{q}, \mathrm{d}, J=100.6 \mathrm{~Hz}), 131.7(\mathrm{~d}, J=2.7 \mathrm{~Hz}), 132.8(\mathrm{~d}, J=9.9 \mathrm{~Hz})$, $133.48(\mathrm{~d}, J=1.6 \mathrm{~Hz}), 154.6(\mathrm{q}) ;{ }^{31} \mathrm{P} \mathrm{NMR}\left(\mathrm{CDCl}_{3}, \mathrm{H}_{3} \mathrm{PO}_{4}, 121.4 \mathrm{MHz}\right) \delta 0.89 ; \mathrm{MS}$ (EI, 70 eV) $m / z$ (rel int) $752\left(\mathrm{M}^{+}, 22\right), 262$ (100). Anal. Calcd for $\mathrm{C}_{52} \mathrm{H}_{38} \mathrm{~N}_{2} \mathrm{P}_{2}$ (752.83): C, 82.96; H, 5.09; N, 3.72. Found: C, 82.77; H, 5.02; N, 3.60.

Bis(iminophosphorane) 9b $\left(\mathbf{R}^{\mathbf{1}}=\mathbf{C H}_{3}\right)$ : Yield 95\%; mp $131{ }^{\circ} \mathrm{C}$ (colorless prisms, acetonitrile); IR (Nujol) 2196, 2127, 1599, 1547, 1440, 1265, 1183, 1107, 1038, 1022, 997, 882, 811, 749, 716, $694 \mathrm{~cm}^{-1} ;{ }^{1} \mathrm{H}$ NMR $\left(\mathrm{CDCl}_{3}, 300 \mathrm{MHz}\right) \delta 2.16(\mathrm{~s}, 6 \mathrm{H}), 6.38(\mathrm{dd}, 2 \mathrm{H}, J=$ 8.2, 1.1 Hz), 6.68 (dd, 2 H, $J=8.2,1.9$ Hz), 7.23-7.24 (m, 2 H), 7.36-7.40 (m, 18 H), 7.79$7.88(\mathrm{~m}, 12 \mathrm{H}) ;{ }^{13} \mathrm{C} \mathrm{NMR}\left(\mathrm{CDCl}_{3}, 75 \mathrm{MHz}\right) \delta 20.4,78.0(\mathrm{q}), 83.2$ (q), 117.9 (q, d, $J=22.3$ Hz), 121.2 (d, $J=8.9 \mathrm{~Hz}), 126.0$ (q), 128.6 (d, $J=12.1 \mathrm{~Hz}), 129.9,130.9$ (q, d, $J=100.3 \mathrm{~Hz})$, $131.5(\mathrm{~d}, J=2.7 \mathrm{~Hz}), 132.8(\mathrm{~d}, J=9.9 \mathrm{~Hz}), 133.7,152.1(\mathrm{q}) ;{ }^{31} \mathrm{P} \mathrm{NMR}\left(\mathrm{CDCl}_{3}, \mathrm{H}_{3} \mathrm{PO}_{4}, 121.4\right.$ MHz) $\delta$ 0.25; MS (EI, $70 \mathrm{eV}) \mathrm{m} / \mathrm{z}$ (rel int) $780\left(\mathrm{M}^{+}, 6\right), 183(100)$. Anal. Calcd for $\mathrm{C}_{54} \mathrm{H}_{42} \mathrm{~N}_{2} \mathrm{P}_{2}$ (780.89): C, 83.06; H, 5.42; N, 3.59. Found: C, 82.87; H, 5.32; N, 3.55.

\section{Analytical and Spectroscopic Data for Bis(benzocarbazole) 10b}

Bis(benzocarbazole) 10b $\left(\mathbf{R}^{1}=\mathbf{C H}_{3}\right)$ : eluent for column chromatography, hexanes/ethyl acetate $\left(4: 1, \mathrm{v} / \mathrm{v}\right.$ ); Yield 96\%; $\mathrm{mp}>320{ }^{\circ} \mathrm{C}$ (colorless prisms); IR (Nujol) 3447, 3295, 1613, 1599, 1338, 1293, 1259, 1230, 1154, 1146, 1129, 1107, 1070, 946, 807, 758, $702 \mathrm{~cm}^{-1}$; ${ }^{1} \mathrm{H}$ NMR $\left(\mathrm{CDCl}_{3}, 400 \mathrm{MHz}\right) \delta 1.89(\mathrm{~s}, 6 \mathrm{H}), 5.85(\mathrm{~s}, 2 \mathrm{H}), 6.95(\mathrm{dd}, 2 \mathrm{H}, J=8.1,1.2 \mathrm{~Hz}), 7.07$ (d, $2 \mathrm{H}, J=8.1 \mathrm{~Hz}), 7.11-7.15$ (m, $2 \mathrm{H}), 7.39$ (ddd, $2 \mathrm{H}, J=8.6,6.6,1.3 \mathrm{~Hz}), 7.58-7.64$ (m, 4 $\mathrm{H}), 7.72(\mathrm{t}, 4 \mathrm{H}, J=7.7 \mathrm{~Hz}), 7.77-7.83(\mathrm{~m}, 6 \mathrm{H}), 8.00(\mathrm{~d}, 2 \mathrm{H}, J=8.6 \mathrm{~Hz}) ;{ }^{13} \mathrm{C} \mathrm{NMR}\left(\mathrm{CDCl}_{3}\right.$, $100 \mathrm{MHz}) \delta 21.4,1094,118.3$ (q), 123.0, 123.3, 123.5 (q), 123.9 (q), 124.6, 125.3, 126.6, 127.9 (q), 128.0, 128.1, 128.3 (q), 129.4, 129.5, 129.6 (q), 130.9 (q), 131.0, 131.4, 137.3 (q), 138.1 (q), 140.3 (q); HRMS (EI): m/z: calcd for $\mathrm{C}_{46} \mathrm{H}_{32} \mathrm{~N}_{2}$ : 612.2565; found: 612.2568. 


\section{Analytical and Spectroscopic Data of Bis(quinindolines) 11b-d}

Bis(quinindoline) 11b $\left(\mathbf{R}^{1}=\mathbf{H}, \mathbf{R}^{2}=\mathbf{O C H}_{3}\right)$ : Yield $80 \%$; $\mathrm{mp}>320^{\circ} \mathrm{C}$ (colorless prisms); IR (Nujol) 3444, 3329, 1631, 1609, 1579, 1515, 1486, 1344, 1260, 1234, 1173, 1032, 827, 767, $745 \mathrm{~cm}^{-1} ;{ }^{1} \mathrm{H} \mathrm{NMR}\left(\mathrm{CDCl}_{3}, 300 \mathrm{MHz}\right) \delta 3.35(\mathrm{~s}, 6 \mathrm{H}), 6.13(\mathrm{~d}, 2 \mathrm{H}, J=7.8 \mathrm{~Hz}), 6.58-6.63$ (m, 4 H), 7.24-7.30 (m, 2 H), 7.43-7.49 (m, 4 H), 8.16 (d, 2 H, J = 9.2 Hz), $11.93(\mathrm{~s}, 2 \mathrm{H}) ;{ }^{13} \mathrm{C}$ NMR $\left(\mathrm{CDCl}_{3}, 75 \mathrm{MHz}\right) \delta 55.1,103.6,111.0,115.6$ (q), 119.3 (q), 119.4, 120.9, 121.7, 122.4 (q), 128.1, 129.3, 133.7 (q), 141.8 (q), 142.4 (q), 151.7 (q), 155.2 (q); HRMS (EI): m/z: calcd for $\mathrm{C}_{32} \mathrm{H}_{22} \mathrm{~N}_{4} \mathrm{O}_{2}$ : 494.1743; found: 494.1745 .

Bis(quinindoline) 11c $\left(\mathbf{R}^{\mathbf{1}}=\mathbf{C H}_{3}, \mathbf{R}^{\mathbf{2}}=\mathbf{C H}_{3}\right)$ : Yield $76 \% ; \mathrm{mp}>320{ }^{\circ} \mathrm{C}$ (colorless prisms); IR (Nujol) 3405, 3141, 1612, 1486, 1359, 1341, 1295, 1248, 1229, 1217, 1153, 1129, 818, 798, 727, $637 \mathrm{~cm}^{-1}$; ${ }^{1} \mathrm{H}$ NMR (DMSO-d $\left.d_{6}, 300 \mathrm{MHz}\right) \delta 1.78(\mathrm{~s}, 6 \mathrm{H}), 2.14(\mathrm{~s}, 6 \mathrm{H}), 5.83(\mathrm{~s}, 2 \mathrm{H})$, 6.98-6.99 (m, 2 H), 7.08 (dd, 2 H, $J=8.3,1.4 \mathrm{~Hz}), 7.33$ (d, $2 \mathrm{H}, J=8.2 \mathrm{~Hz}), 7.54(\mathrm{dd}, 2 \mathrm{H}, J$ $=8.9,1.9 \mathrm{~Hz}), 8.09(\mathrm{~d}, 2 \mathrm{H}, J=8.6 \mathrm{~Hz}), 11.82(\mathrm{~s}, 2 \mathrm{H}) ;{ }^{13} \mathrm{C} \mathrm{NMR}\left(\mathrm{DMSO}-d_{6}, 75 \mathrm{MHz}\right) \delta$ 20.8, 21.0, 110.8, 115.4 (q), 119.7 (q), 121.4, 121.9 (q), 123.4, .127.6, 127.9 (q), 129.2, 131.3, 132.5 (q), 134.5 (q), 139.9 (q), 145.0 (q), 152.5 (q); HRMS (EI): m/z: calcd for $\mathrm{C}_{34} \mathrm{H}_{26} \mathrm{~N}_{4}$ : 490.2158; found: 490.2159 .

Bis(quinindoline) 11d $\left(\mathbf{R}^{\mathbf{1}}=\mathbf{C H}_{3}, \mathbf{R}^{\mathbf{2}}=\mathbf{O C H}_{3}\right.$ ): Yield 98\%; $\mathrm{mp}>320{ }^{\circ} \mathrm{C}$ (yellow prisms); IR (Nujol) 3148, 1620, 1515, 1339, 1293, 1258, 1236, 1218, 1179, 1131, 1119, 1034, 822, 798, 754, 745, $727 \mathrm{~cm}^{-1} ;{ }^{1} \mathrm{H}$ NMR (DMSO- $\left.d_{6}, 400 \mathrm{MHz}\right) \delta 1.80(\mathrm{~s}, 6 \mathrm{H}), 3.35$ (s, $\left.6 \mathrm{H}\right), 5.87$ (s, 2 H), $6.57(\mathrm{~d}, 2 \mathrm{H}, \mathrm{J}=2.8 \mathrm{~Hz}), 7.08-7.11(\mathrm{~m}, 2 \mathrm{H}), 7.33(\mathrm{~d}, 2 \mathrm{H}, \mathrm{J}=8.2 \mathrm{~Hz}), 7.46(\mathrm{dd}, 2 \mathrm{H}, \mathrm{J}=$ 9.2, $2.8 \mathrm{~Hz}), 8.14(\mathrm{~d}, 2 \mathrm{H}, \mathrm{J}=9.2 \mathrm{~Hz}), 11.76$ (s, $2 \mathrm{H}$ ); ${ }^{13} \mathrm{C}$ NMR (DMSO-d $\left.6,100 \mathrm{MHz}\right) \delta 20.8$, 55.1, 103.6, 110.7, 115.5 (q), 119.4 (q), 120.8, 121.6, 122.3 (q), 127.7 (q), 129.2, 129.3, 133.6 (q), 139.9 (q), 142.3 (q), 151.8 (q), 155.1 (q); HRMS (EI): m/z: calcd for $\mathrm{C}_{34} \mathrm{H}_{26} \mathrm{~N}_{4} \mathrm{O}_{2}$ : 522.2056; found: 522.2058 .

\section{Preparation of iminophosphorane 12}

To a solution of bis(iminophosphorane) 9a $(1.13 \mathrm{~g}, 1.5 \mathrm{mmol})$ in anhydrous toluene $(100 \mathrm{~mL})$ at $50{ }^{\circ} \mathrm{C}$ a solution of diphenylketene $(0.29 \mathrm{~g}, 1.5 \mathrm{mmol})$ in the same solvent $(10 \mathrm{~mL})$ was added using a syringe pump in about $6 \mathrm{~h}$. The stirring was continued at $50{ }^{\circ} \mathrm{C}$ for $2 \mathrm{~h}$. The 
solvent was removed under reduced pressure, and the resulting material was purified by silica gel column chromatography using hexanes/diethyl ether $(3: 7, \mathrm{v} / \mathrm{v})$ as eluent.

Iminophosphorane 12: Yield 54\%; mp $133{ }^{\circ} \mathrm{C}$ (yellow prisms, diethyl ether); IR (Nujol) 3424, 2187, 1605, 1585, 1434, 1343, 1274, 1184, 1153, 1106, 1072, 1053, 1025, 750, 717, $694 \mathrm{~cm}^{-1} ;{ }^{1} \mathrm{H} \mathrm{NMR}\left(\mathrm{CDCl}_{3}, 400 \mathrm{MHz}\right) \delta 6.58(\mathrm{~d}, 1 \mathrm{H}, J=8.1 \mathrm{~Hz}), 6.76(\mathrm{t}, 1 \mathrm{H}, J=7.4 \mathrm{~Hz})$, 6.92-6.96 (m, 2 H), 7.14-7.43 (m, 4 H), 7.49-7.54 (m, 1 H), 7.59-7.61 (m, 4 H), 7.74 (dt, 1 H, $J=7.5,1.9 \mathrm{~Hz}), 7.79-7.89(\mathrm{~m}, 5 \mathrm{H}), 8.96(\mathrm{~d}, 1 \mathrm{H}, J=8.3 \mathrm{~Hz}), 9.16(\mathrm{~d}, 1 \mathrm{H}, J=7.8 \mathrm{~Hz}) ;{ }^{13} \mathrm{C}$ $\operatorname{NMR}\left(\mathrm{CDCl}_{3}, 100 \mathrm{MHz}\right) \delta 89.7$ (q), 102.0 (q), 109.5, 114.4 (q), 117.5, 118.1 (q), 119.2 (q, d, $J=22.8 \mathrm{~Hz}), 119.5,121.7(\mathrm{~d}, J=9.5 \mathrm{~Hz}), 123.2,123.8,124.2,124.5,125.2$ (s), 125.3, 127.4, 127.6, 127.9, 128.6 (d, $J=12.2 \mathrm{~Hz}), 128.9,129.3,129.5$ (q), 130.5 (q), 130.8 (q, d, $J=99.7$ Hz), 130.9, 131.4 (q), 131.7 (d, $J=2.6 \mathrm{~Hz}), 132.9$ (d, $J=9.9 \mathrm{~Hz}), 133.5,136.8,137.3$ (q), 141.9 (q), 153.9 (q); ${ }^{31} \mathrm{P}$ NMR $\left(\mathrm{CDCl}_{3}, \mathrm{H}_{3} \mathrm{PO}_{4}, 121.4 \mathrm{MHz}\right) \delta 2.03$; MS (EI, $\left.70 \mathrm{eV}\right) \mathrm{m} / z$ (rel int) $668\left(\mathrm{M}^{+}, 48\right), 262$ (100). Anal. Calcd for $\mathrm{C}_{48} \mathrm{H}_{33} \mathrm{~N}_{2} \mathrm{P}$ (668.78): C, 86.21; H, 4.97; N, 4.19. Found: C, 86.03; H, 5.07; N, 4.07.

\section{Preparation of Benzocarbazole-Quinindolines 13}

To a solution of iminophosphorane $12(0.67 \mathrm{~g}, 1 \mathrm{mmol})$ in anhydrous toluene $(20 \mathrm{~mL}) \mathrm{a}$ solution of the arylisocyanate $(2 \mathrm{mmol})$ in the same solvent $(5 \mathrm{~mL})$ was added. The reaction mixture was stirred at room temperature for $12 \mathrm{~h}$. The toluene was removed under reduced pressure and the residue was chromatographed on a silica gel column, using hexanes/diethyl ether as eluent.

Benzocarbazole-Quinindoline 13a $\left(\mathbf{R}^{2}=\mathbf{C H}_{3}\right)$ : eluent for column chromatography, hexanes/diethyl ether (3:2, v/v); Yield 77\%; mp $>320{ }^{\circ} \mathrm{C}$ (yellow prisms); IR (Nujol) 3345, 1706, 1622, 1608, 1585, 1560, 1345, 1314, 1294, 1271, 1258, 1234, 1178, 1109, 1085, 822, 760, 740, 714, $704 \mathrm{~cm}^{-1} ;{ }^{1} \mathrm{H}$ NMR $\left(\mathrm{CDCl}_{3}, 400 \mathrm{MHz}\right) \delta 2.27$ (s, $\left.3 \mathrm{H}\right), 2.42$ (s, $\left.3 \mathrm{H}\right), 6.18$ (dd, $1 \mathrm{H}, J=7.8,0.5 \mathrm{~Hz}), 6.33(\mathrm{~d}, 1 \mathrm{H}, J=8.0 \mathrm{~Hz}), 6.60$ (ddd, $1 \mathrm{H}, J=8.1,6.7,1.5 \mathrm{~Hz}), 6.71(\mathrm{td}$, $1 \mathrm{H}, J=8.0,0.9 \mathrm{~Hz}), 7.13-7.21(\mathrm{~m}, 3 \mathrm{H}), 7.27-7.30(\mathrm{~m}, 4 \mathrm{H}), 7.40-7.43(\mathrm{~m}, 2 \mathrm{H}), 7.59-7.64$ (m, 2 H), 7.68-7.72 (m, 2 H), 7.78-7.83 (m, 4 H), 8.01-8.04 (m, 1 H), 8.09 (s, 1 H), 8.26 (d, 1 $\mathrm{H}, J=8.6 \mathrm{~Hz}), 8.79(\mathrm{~d}, 1 \mathrm{H}, J=8.3 \mathrm{~Hz}), 13.23(\mathrm{~s}, 1 \mathrm{H}) ;{ }^{13} \mathrm{C} \mathrm{NMR}\left(\mathrm{CDCl}_{3}, 100 \mathrm{MHz}\right) \delta 21.0$, $21.7,110.2,116.8,119.0$ (q), 119.5 (q), 119.7, 120.6, 121.7 (q), 122.3, 122.4 (q), 122.6, 122.9 (q), 123.3, 123.7, 125.0, 125.2 (q), 125.4 (q), 125.5, 125.7, 126.7 (q), 127.5, 127.6, 128.3, 
128.9, 129.5, 129.8, 130.8 (q), 130.9, 131.2, 132.6, 133.6 (q), 135.7 (q), 136.0 (q), 136.5 (q), 137.5 (q), 139.9 (q), 140.7 (q), 142.2 (q), 143.1 (q), 150.8 (q), 151.2 (q); HRMS (EI): m/z: calcd for $\mathrm{C}_{46} \mathrm{H}_{32} \mathrm{~N}_{4} \mathrm{O}$ : 656.2576 ; found: 656.2579 .

Benzocarbazole-Quinindoline 13b $\left(\mathbf{R}^{2}=\mathbf{O C H}_{3}\right)$ : eluent for column chromatography, hexanes/diethyl ether (7:3, v/v); Yield 49\%; mp >320 ${ }^{\circ} \mathrm{C}$ (yellow prisms); IR (Nujol) 3358, $1635,1613,1343,1258,1235,1172,1152,1129,1036,817,772,742,727 \mathrm{~cm}^{-1}$; ${ }^{1} \mathrm{H}$ NMR $\left(\mathrm{DMSO}-d_{6}, 400 \mathrm{MHz}\right) \delta 3.32(\mathrm{~s}, 3 \mathrm{H}), 3.40(\mathrm{~s}, 3 \mathrm{H}), 6.06(\mathrm{~d}, 1 \mathrm{H}, \mathrm{J}=7.8 \mathrm{~Hz}), 6.10(\mathrm{~d}, 1 \mathrm{H}, \mathrm{J}$ $=7.8 \mathrm{~Hz}), 6.52(\mathrm{t}, 1 \mathrm{H}, \mathrm{J}=7.5 \mathrm{~Hz}), 6.58(\mathrm{t}, 1 \mathrm{H}, \mathrm{J}=7.5 \mathrm{~Hz}), 6.66(\mathrm{~d}, 1 \mathrm{H}, \mathrm{J}=2.3 \mathrm{~Hz}), 7.10-$ 7.27 (m, $7 \mathrm{H}), 7.31(\mathrm{~d}, 1 \mathrm{H}, \mathrm{J}=8.5 \mathrm{~Hz}), 7.38-7.43$ (m, $3 \mathrm{H}), 7.49$ (dd, $1 \mathrm{H}, \mathrm{J}=9.2,2.3 \mathrm{~Hz}$ ), 7.62-7.65 (m, $1 \mathrm{H}), 7.74-7.79(\mathrm{~m}, 4 \mathrm{H}), 7.86$ (d, $1 \mathrm{H}, \mathrm{J}=8.7 \mathrm{~Hz}), 8.18$ (d, $1 \mathrm{H}, \mathrm{J}=9.2 \mathrm{~Hz})$, 10.85 (s, $1 \mathrm{H}), 11.83$ (s, $1 \mathrm{H}) ;{ }^{13} \mathrm{C}$ NMR (DMSO- $\left.d_{6}, 100 \mathrm{MHz}\right) \delta 55.1,104.7,110.8,111.2$, 116.8 (q), 118.6, 118.9 (q), 119.3, 120.0 (q), 120.2, 121.2 (q), 121.3, 122.0, 122.4 (q), 123.0, 123.8 (q), 124.6, 125.0, 125.4 (q), 125.6 (q), 127.2, 127.9, 129.2, 129.3, 130.3 (q), 131.1, 131.3, 136.3 (q), 136.8 (q), 137.5 (q), 141.7 (q), 142.6 (q), 143.1 (q), 151.9 (q), 155.0 (q); HRMS (EI): m/z: calcd for $\mathrm{C}_{46} \mathrm{H}_{32} \mathrm{~N}_{4} \mathrm{O}_{3}$ : 688.2474; found: 688.2476 .

\section{Analytical and Spectroscopic Data of Benzocarbazole-Quinindoline 14b}

Benzocarbazole-Quinindoline 14b $\left(\mathbf{R}^{\mathbf{2}}=\mathbf{O C H}_{3}\right)$ : Yield 90\%; mp $317{ }^{\circ} \mathrm{C}$ (yellow prisms); IR (Nujol) 3361, 1635, 1614, 1579, 1521, 1488, 1396, 1342, 1259, 1234, 1174, 1149, 1129, 1107, 1036, 817, 773, 742, 727, $702 \mathrm{~cm}^{-1} ;{ }^{1} \mathrm{H}$ NMR (DMSO-d, $\left.300 \mathrm{MHz}\right) \delta 3.35$ (s, $3 \mathrm{H}$ ); $6.07(\mathrm{~d}, 1 \mathrm{H}, J=7.8 \mathrm{~Hz}), 6.11(\mathrm{~d}, 1 \mathrm{H}, J=7.9 \mathrm{~Hz}), 6.49-6.60(\mathrm{~m}, 2 \mathrm{H}), 6.67(\mathrm{~d}, 1 \mathrm{H}, J=2.8$ Hz), 7.09-7.44 (m, 7 H), 7.49 (dd, $1 \mathrm{H}, J=9.2,2.8 \mathrm{~Hz}), 7.60-7.66$ (m, $1 \mathrm{H}), 7.72-7.78$ (m, 4 H), $7.87(\mathrm{~d}, 1 \mathrm{H}, J=8.6 \mathrm{~Hz}), 8.19(\mathrm{~d}, 1 \mathrm{H}, J=9.2 \mathrm{~Hz}), 10.86(\mathrm{~s}, 1 \mathrm{H}), 11.85(\mathrm{~s}, 1 \mathrm{H}) ;{ }^{13} \mathrm{C}$ NMR (DMSO- $d_{6}, 750$ MHz) $\delta 55.1,104.7,110.7,111.2,116.8$ (q), 118.6, 119.0 (q), 119.3, 119.9 (q), 120.2, 121.3, 121.4 (q), 122.0, 122.4 (q), 123.0, 123.8 (q), 124.6, 125.0, 125.4 (q), 125.6 (q), 127.2, 127.9, 129.2, 130.3 (q), 131.1, 131.3, 136.3 (q), 136.8 (q), 137.5 (q), 141.7 (q), 142.6 (q), 143.2 (q), 151.9 (q), 155.0 (q); HRMS (EI): m/z: calcd for $\mathrm{C}_{38} \mathrm{H}_{25} \mathrm{~N}_{3} \mathrm{O}$ : 539.1998; found: 539.1999 . 
Figure S1. X-Ray structure of 11a

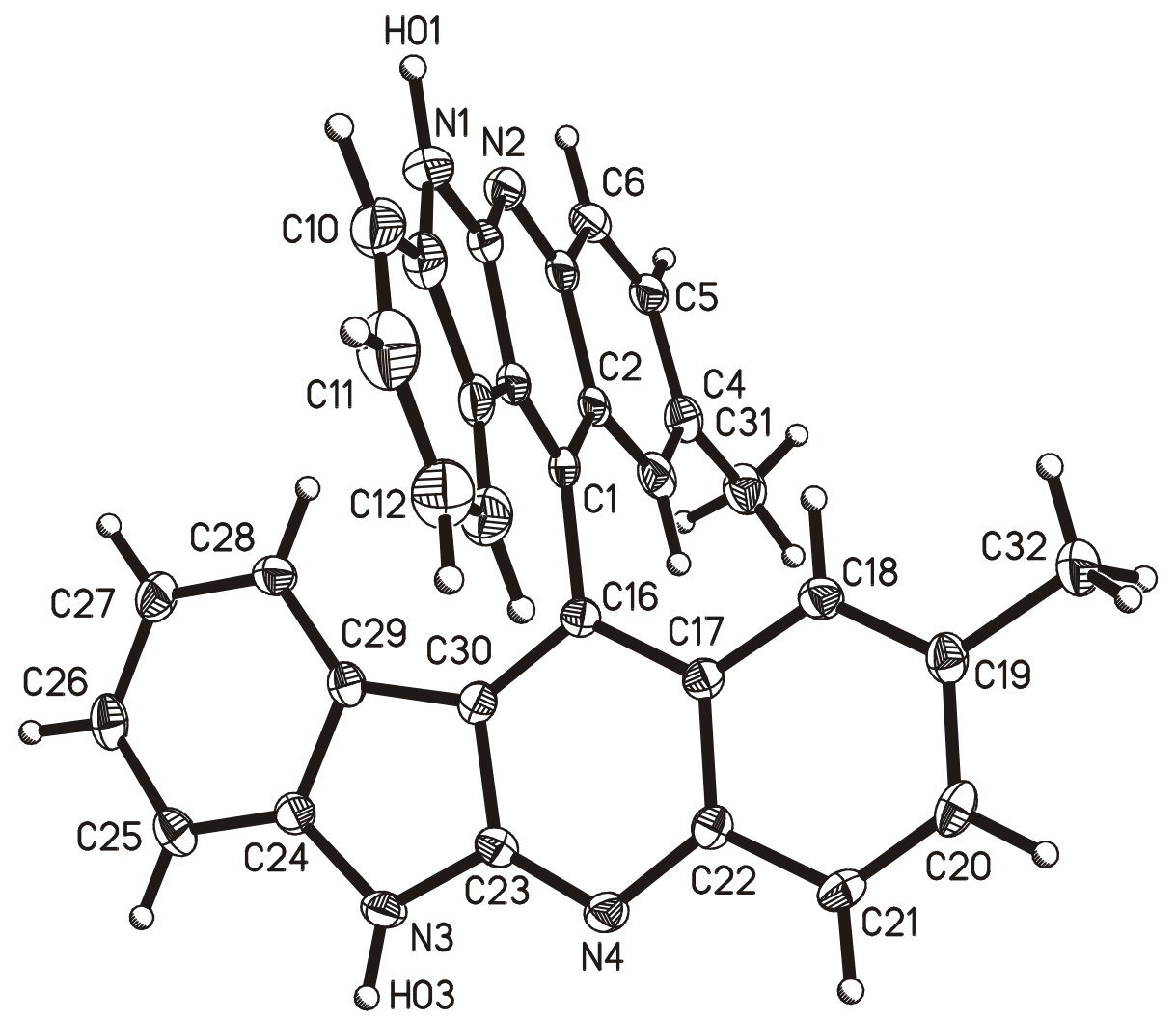

Figure S2. X-Ray structure of 13a

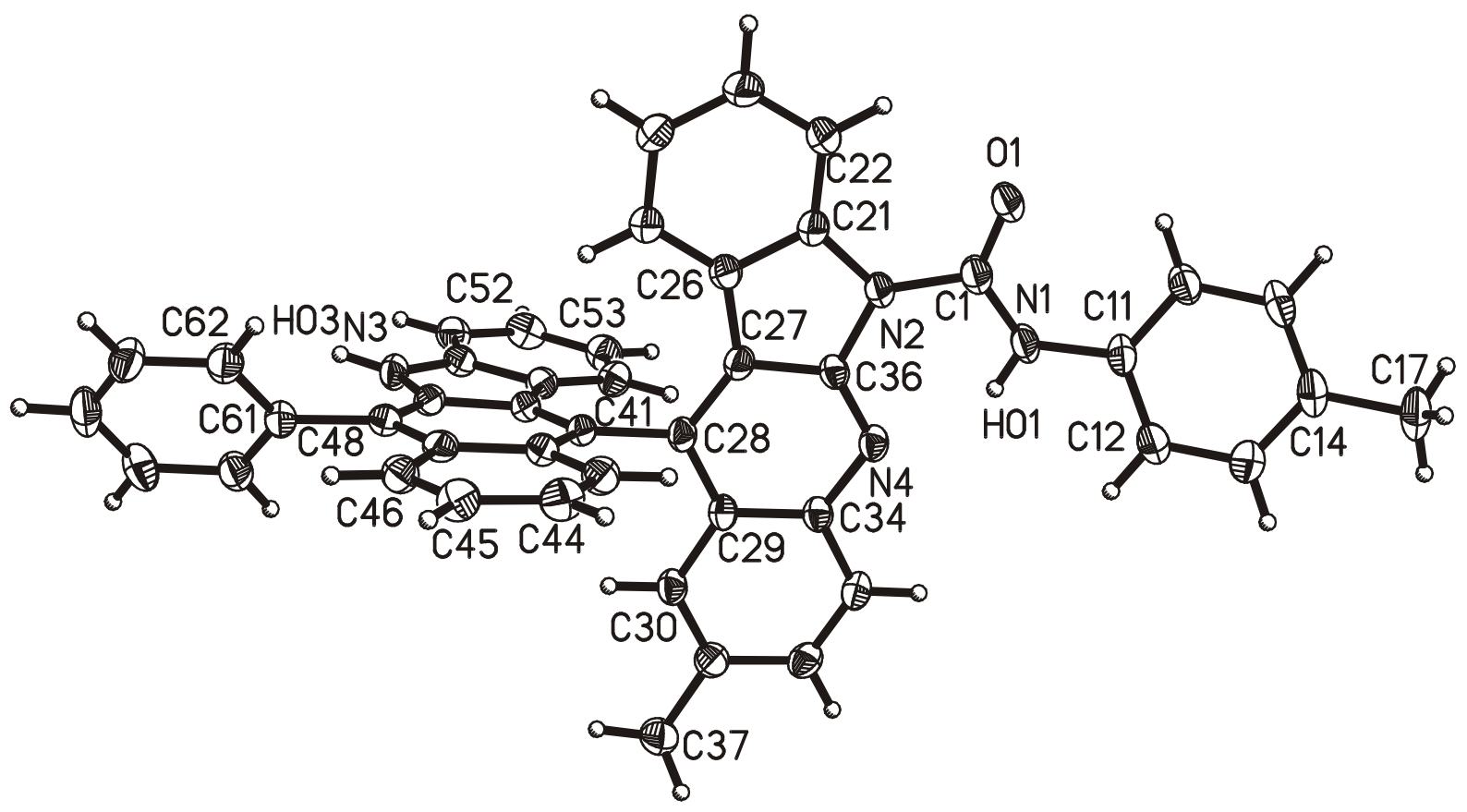




\section{References}

1 Sakai, N.; Annaka, K.; Konakahara, T. J. Org. Chem. 2006, 71, 3653-3655.

2 (a) Saulnier, M. G.; Frennesson, D. B.; Deshpande, M. S.; Vyas, D. M. Tetrahedron Lett. 1995, 36, 7841-7844. (b) Koradin, C.; Dohle, W.; Rodriguez, A. L.; Schmid, N.; Knochel, P. Tetrahedron 2003, 59, 1571-1587.

3 Taylor, E. C.; McKillop, A.; Hawks, G. H. Org. Synth. 1973, 52, 36. 

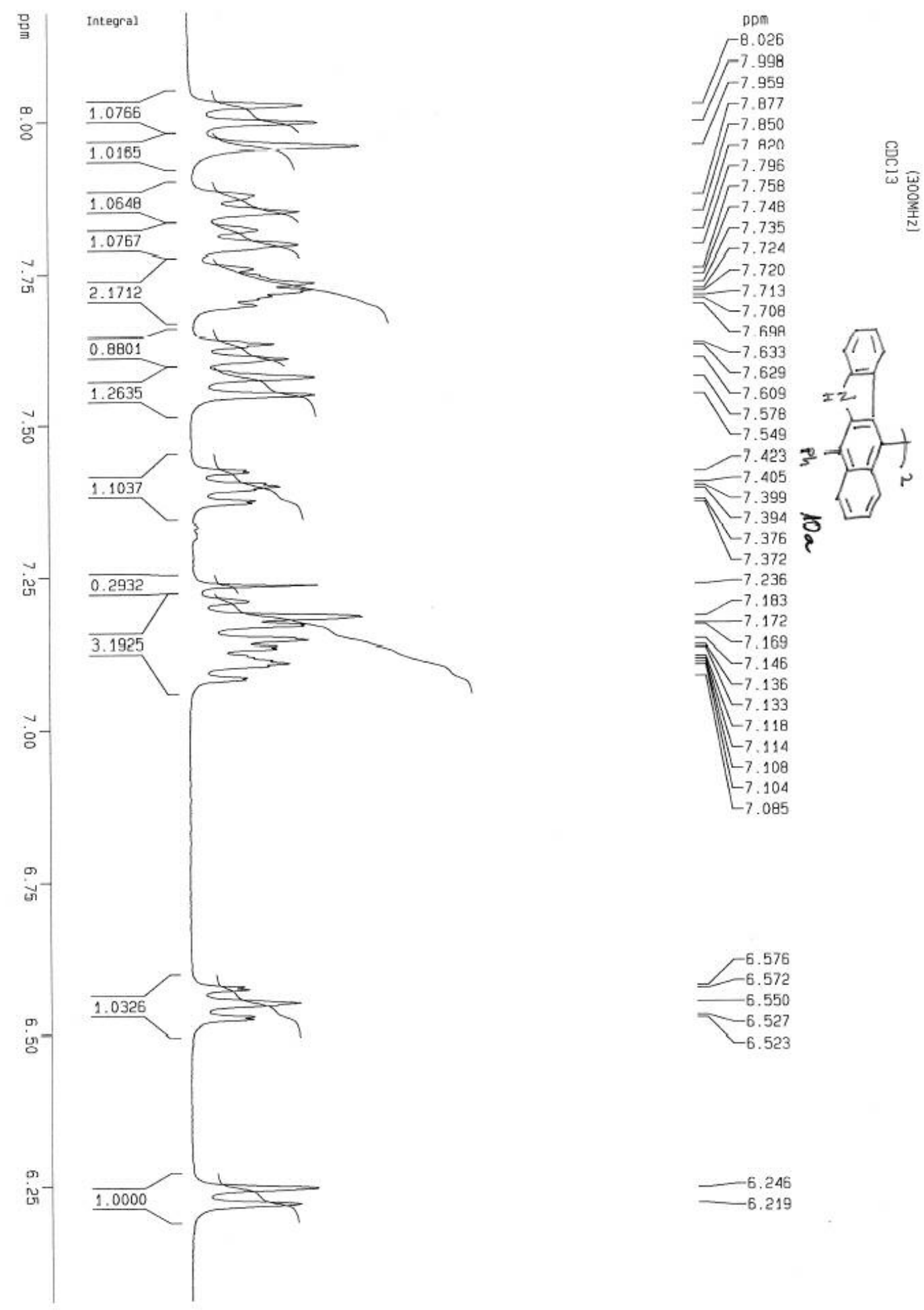

-7.394 z

-7.376 \&

7.372

$-7.236$

$-7.183$

$-7.172$

a -7.169

$-7.146$

$-7.136$

$-7.133$

$-7.118$

$-7.114$

$-7.108$

$-7.104$

$-7.085$

.

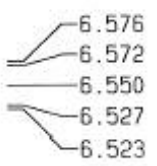

$\begin{array}{r}-6.246 \\ -6.219 \\ \hline\end{array}$ 

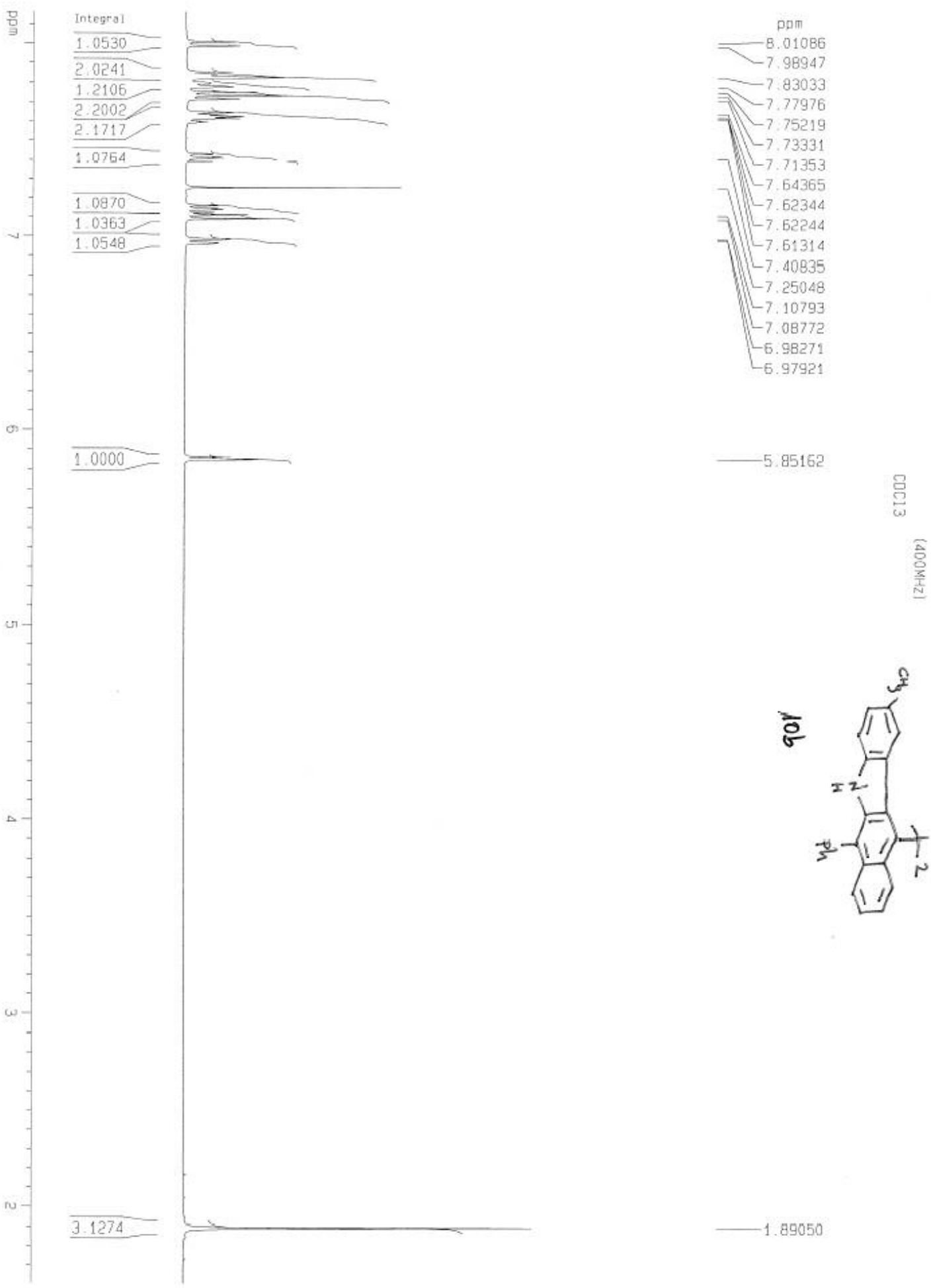


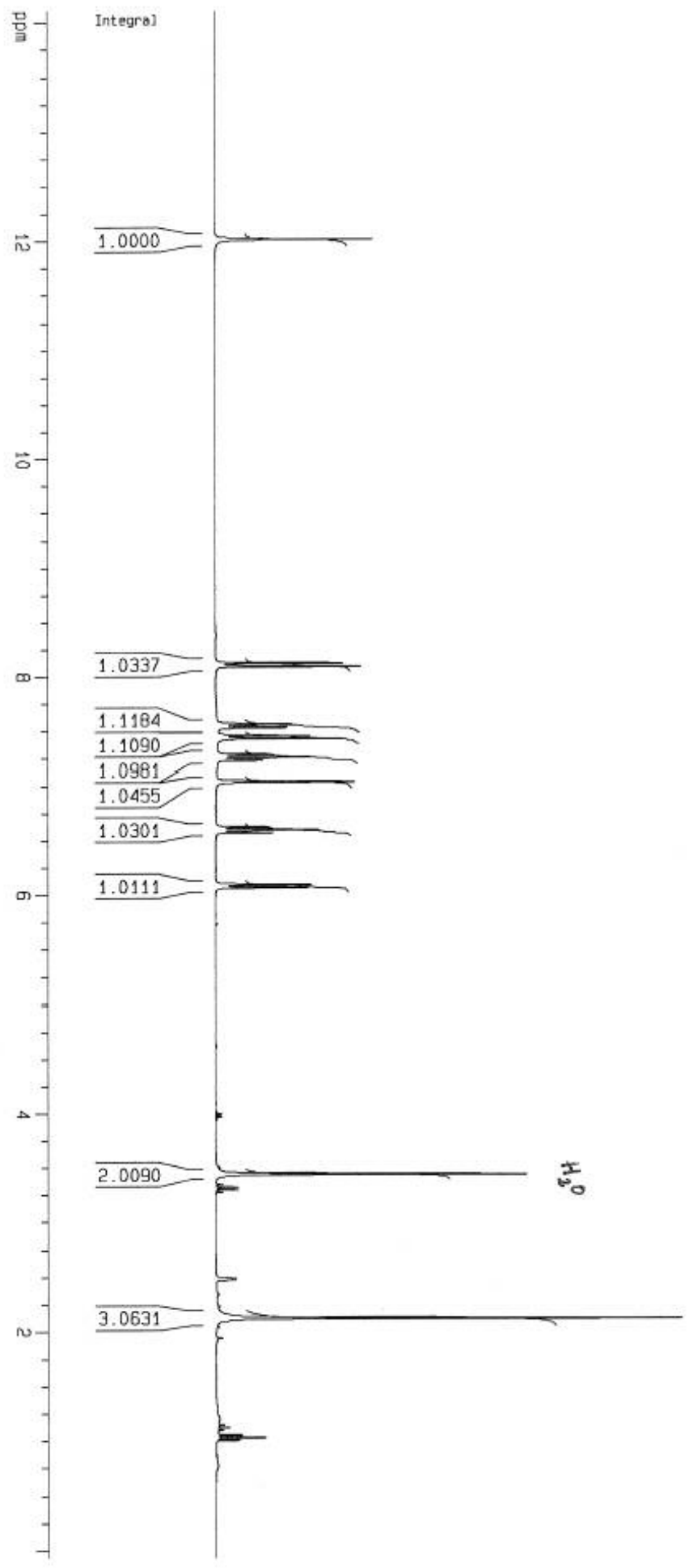

ppm
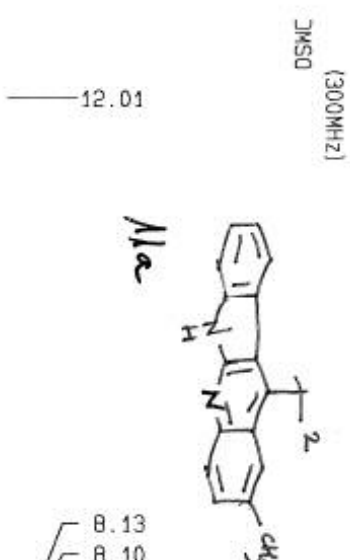

$F^{8.13}$

$7.56^{\circ}$

$\sqrt{-7.53}$

-7.53
-7.46
-7.43

7.43

7. 28

7.26

7.23

7.05

$-6.60$

$-6.58$

6.10

$-6.07$

\section{列}



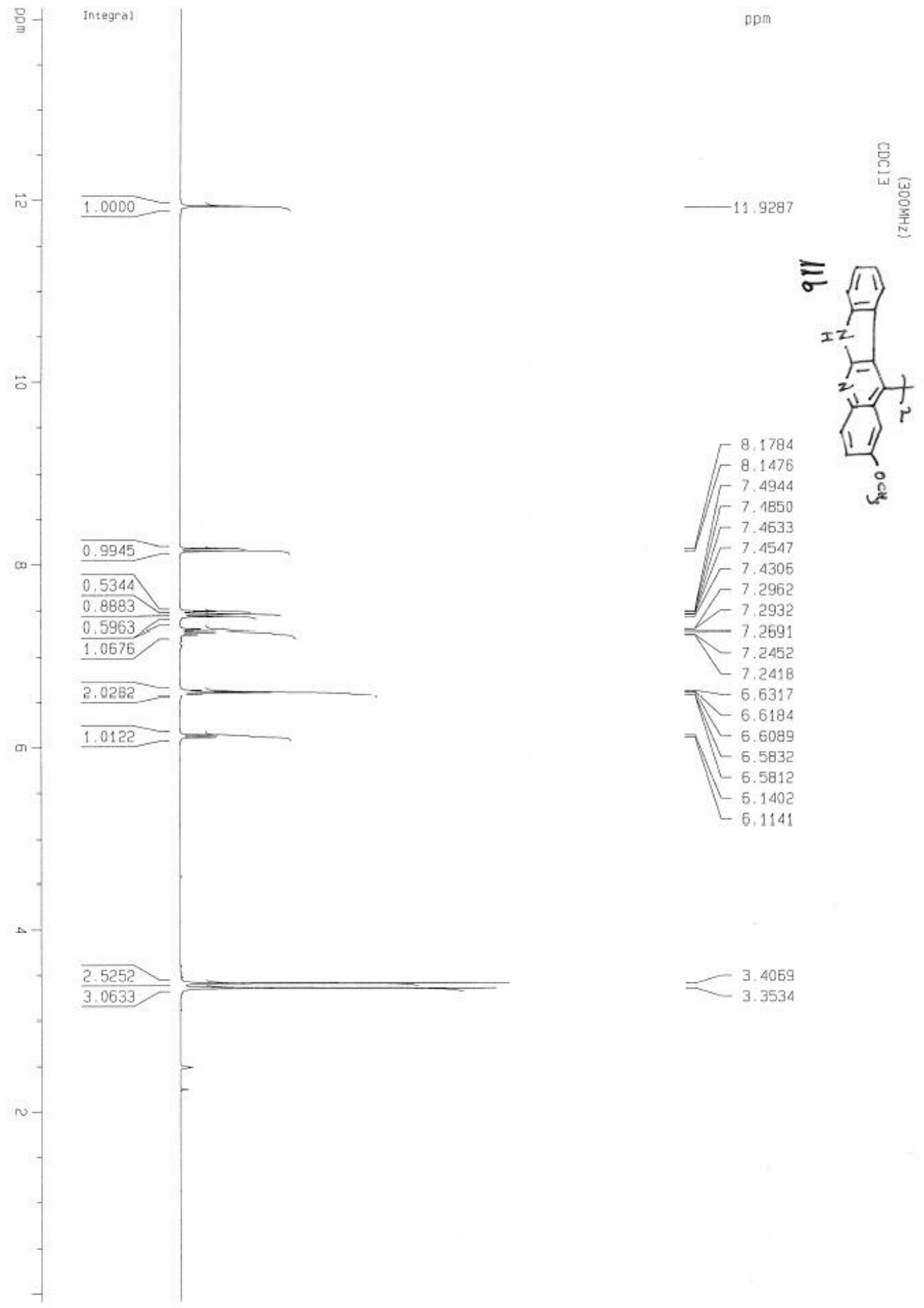

$\left[\begin{array}{r}8,1784 \\ -8,147\end{array}\right.$

- 7.4944

] 7.1850

] 7.4633

I) -7.4547

$\sqrt{-7.4306}$

$\sqrt{-7.2962}$

D -7.2932

$=7.2591$

$-7.2452$

$-7.2418$

$-6.6317$

$-6.6184$

$\leftarrow 6.6089$

$-6.5832$

$-6.5812$

$-6.1402$

$-6.1141$

홍 


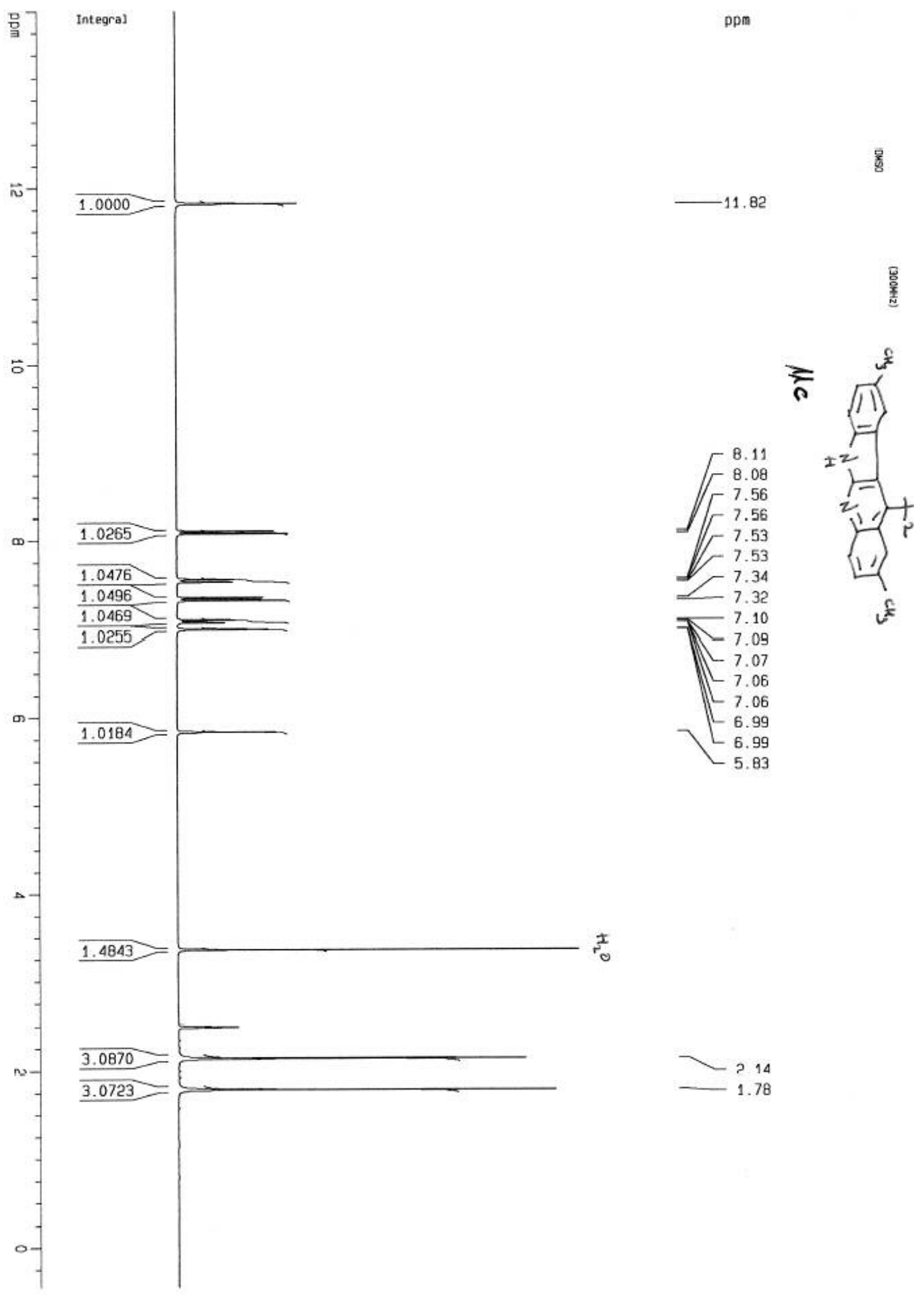




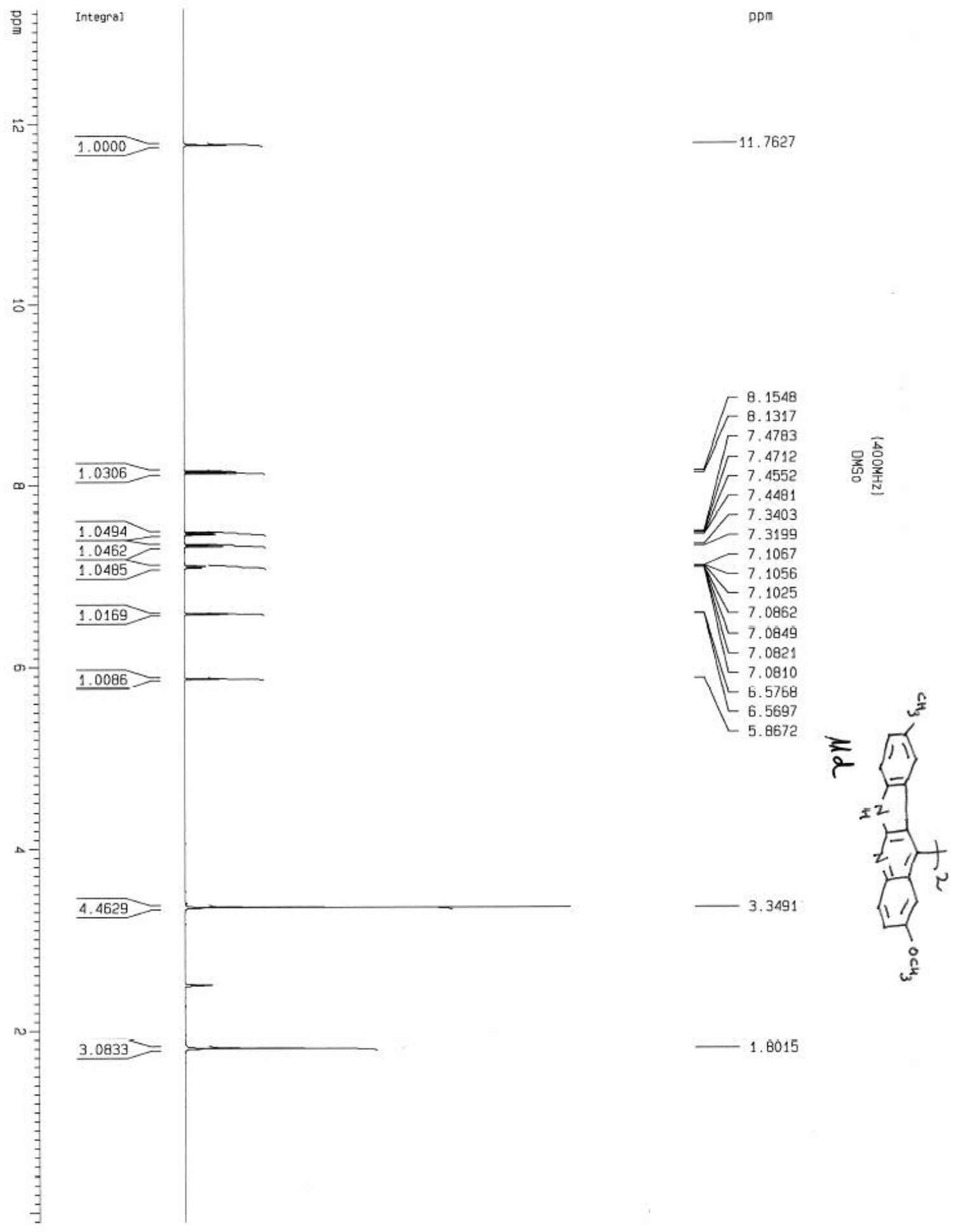



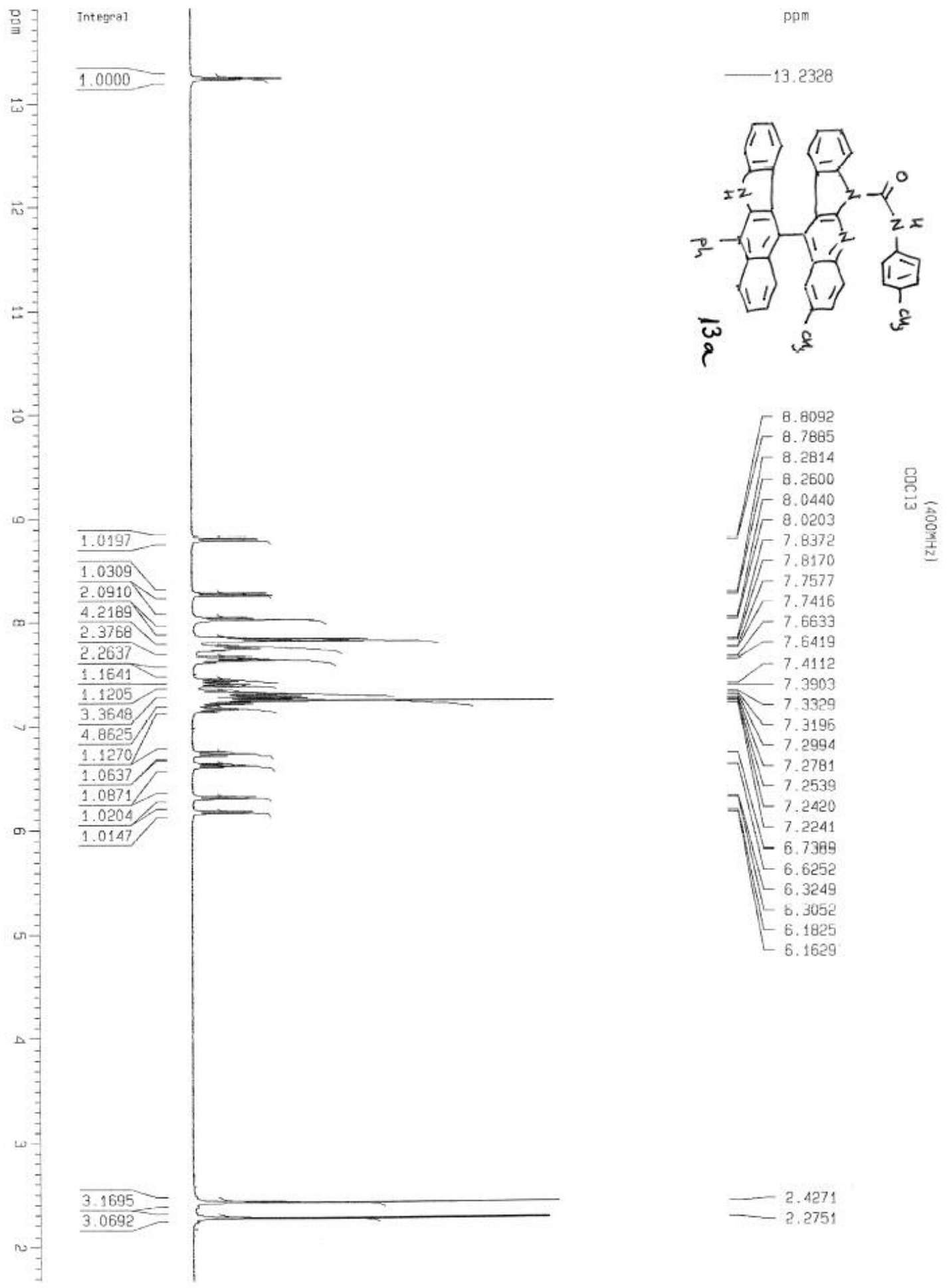

8170

$\int-7.7577$

7.7416

$=\sqrt{7.6633}$

I) -7.6419

7.4112

7.3903

$-7.3329$

$-7.3196$

$-7.2994$

L 7.2781

IIL 7.2539

$-7.2420$

L 7.2241

$-6.7389$

$-6.6252$

$-6.3249$

L b. 3052

- 6.1825

6. 1629

2. 4271

-2.4271
-2.2751 


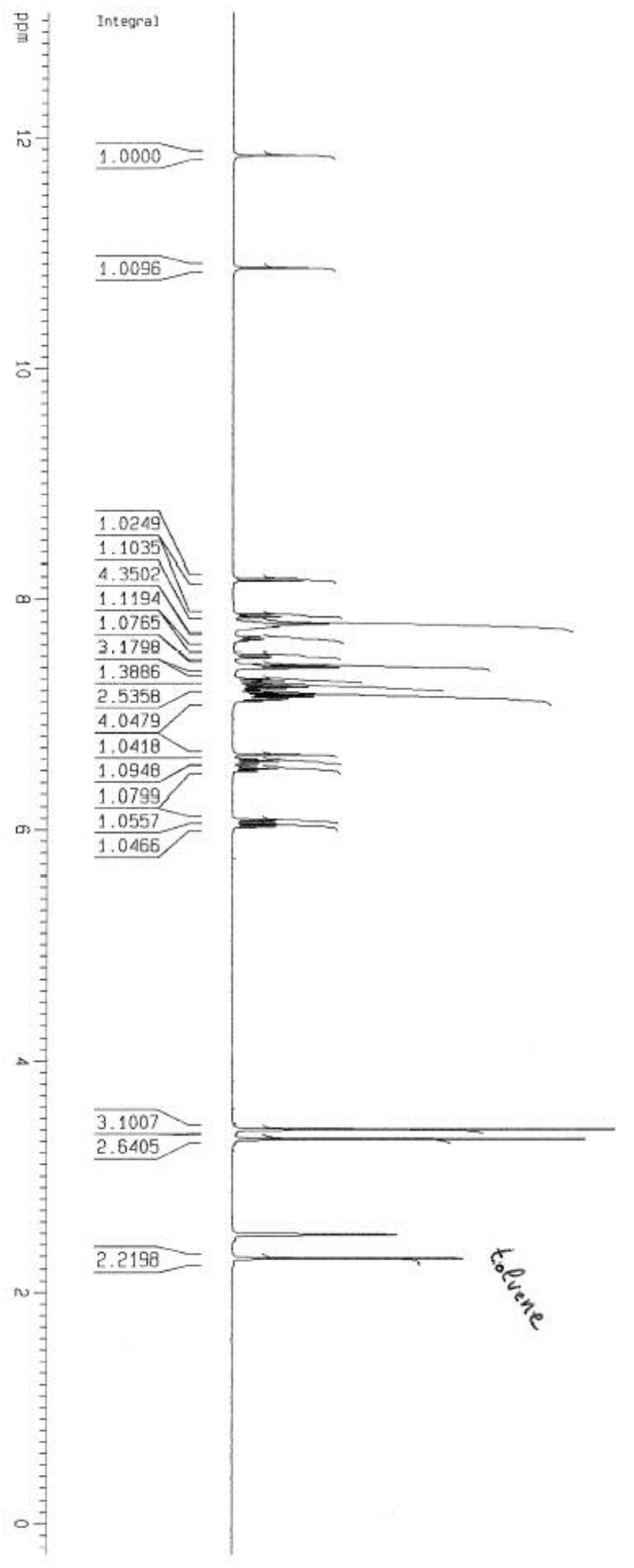

$-7.1467$

$-7.1277$

$-6.6449$

$-6.6379$

- 6.5855

6.5193

$-6.0896$

- 6.0699

- 6.0473

- 6.0278

$-3.3206$

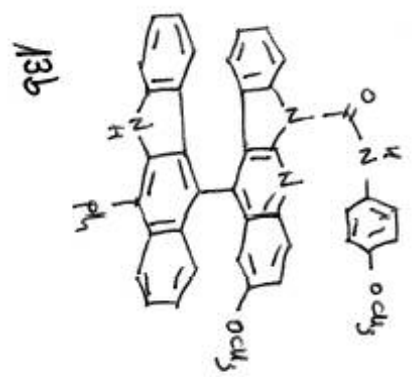



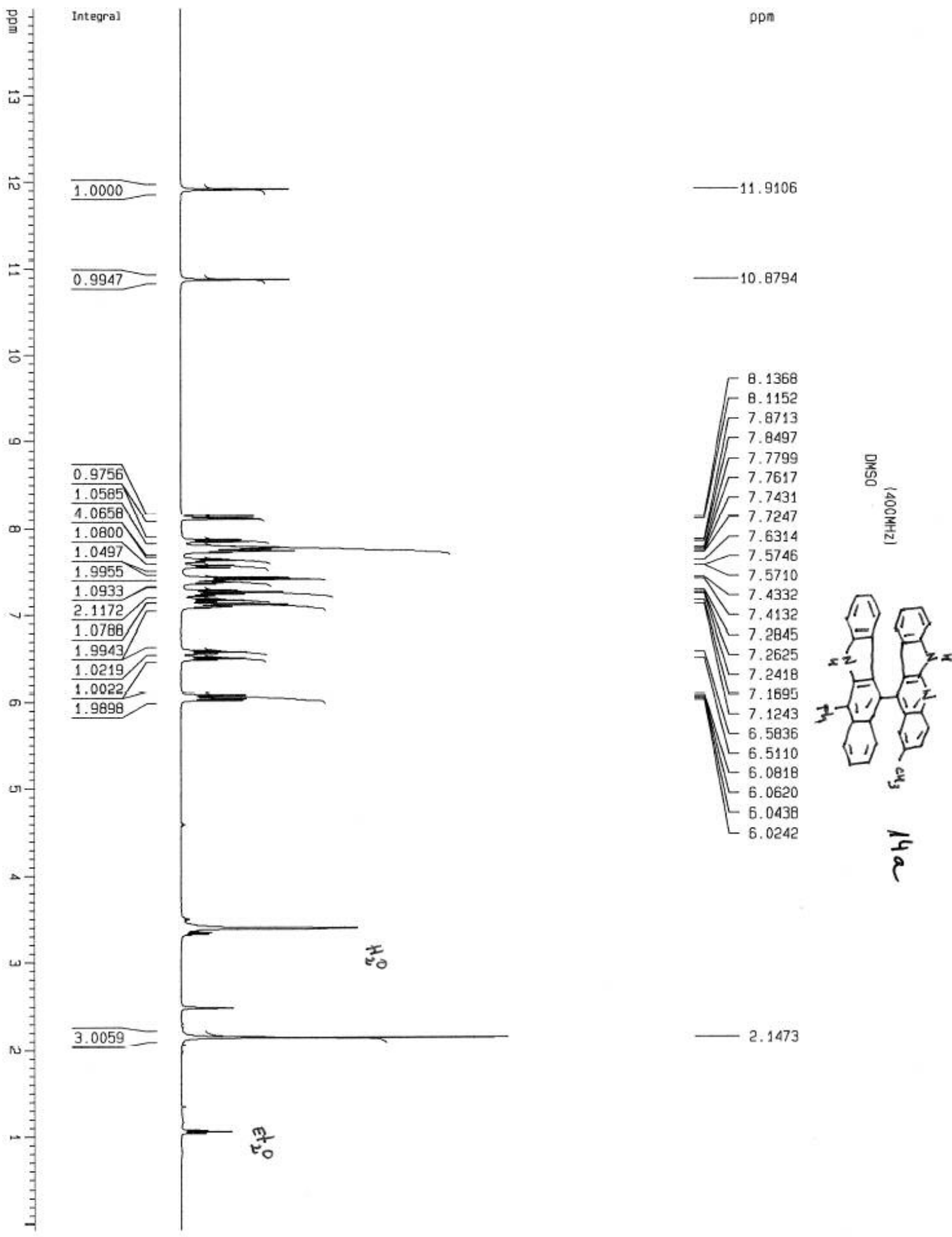

2. 1473 

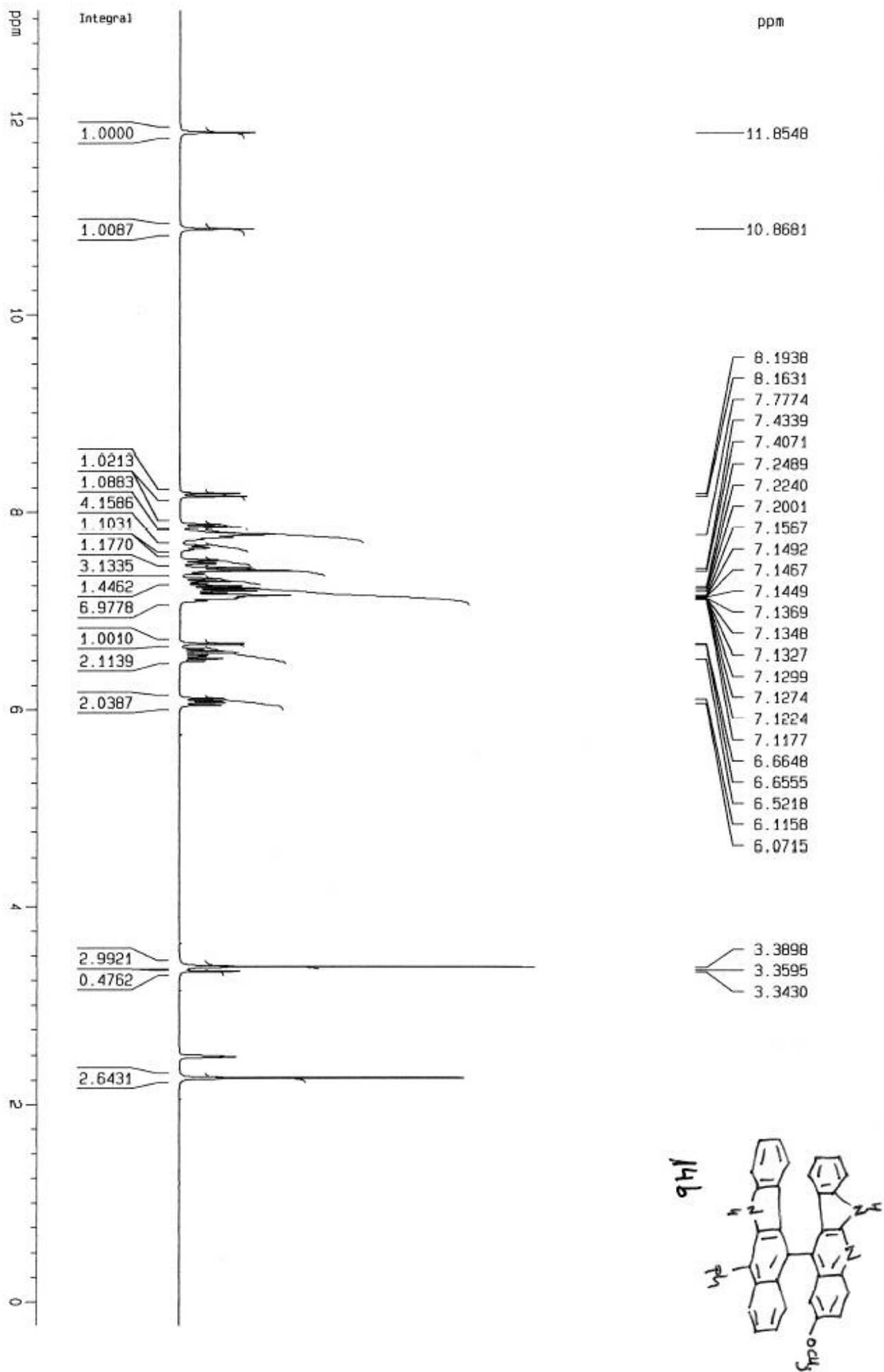

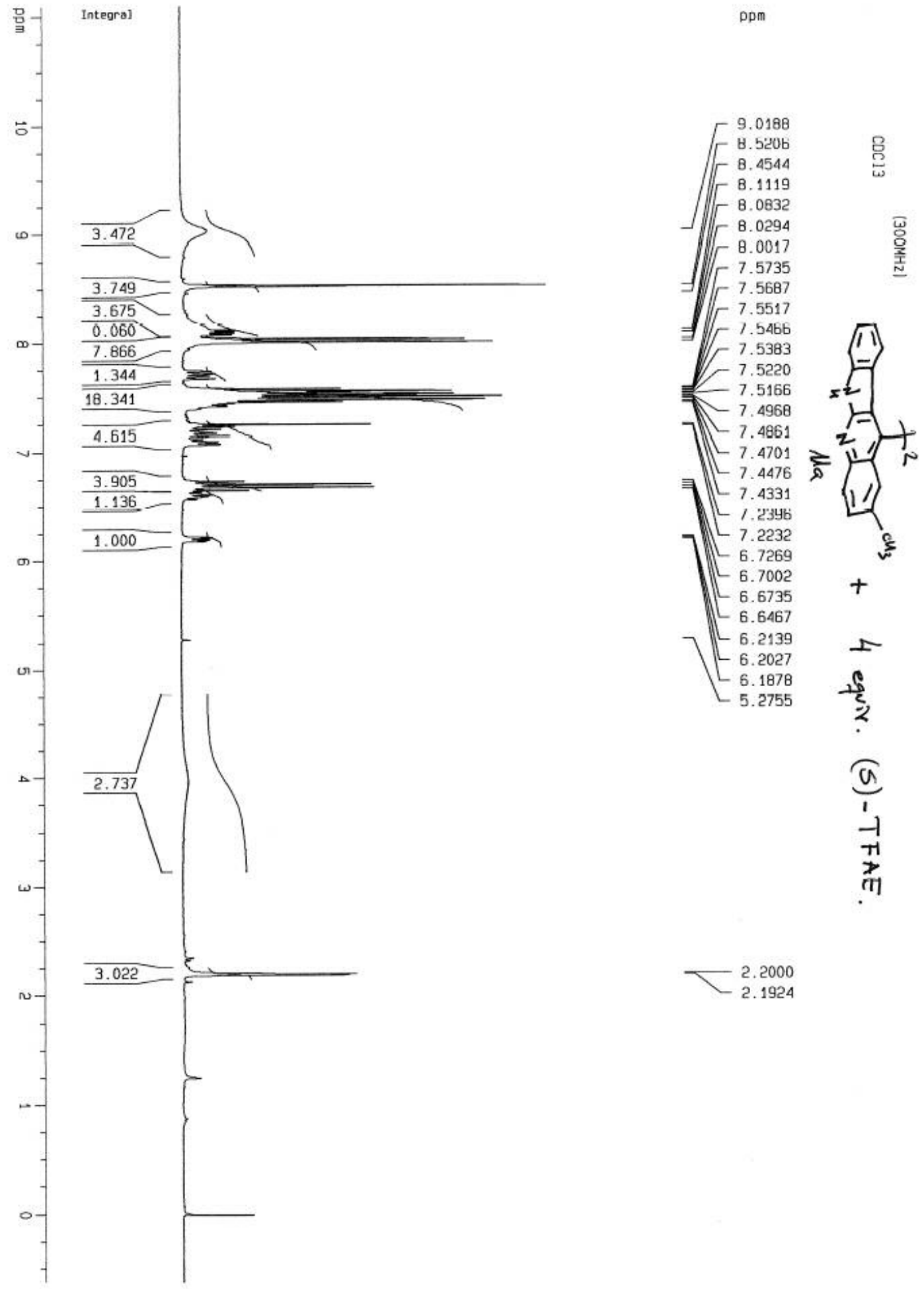

2. 2000

$-2.1924$ 


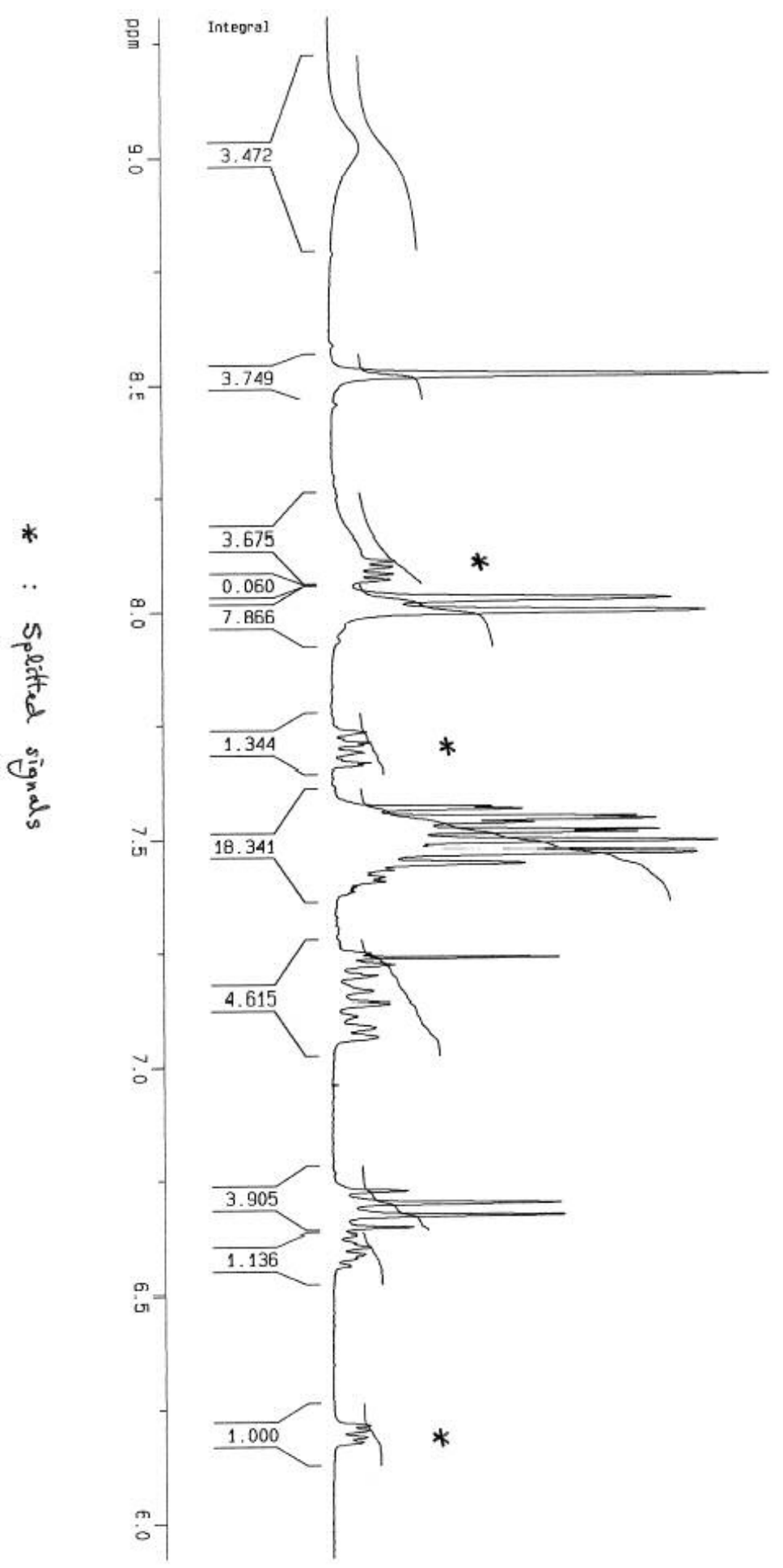

ppm

$-9.01882$

总

$r^{8.520644}$

-8.45444
-8.25233

8.25233
-8.11184

$-8.09929$

$-8.08324$

$\Gamma_{-8.07043}^{8.08324}$

$-8.02938$

8.00174

$-7.73359$

$-7.71096$

-7.66648

-7. $5 / 346$

$-7.56867$

$-7.55165$

$-7.54664$

7.53825

-7.52203

$-7.51662$

-7.49677
-7.48613

$-7.47014$

$-7.44763$

$-7.43306$

$-7.42396$

$7 L_{7.41736}^{-7408}$

$-7.40767$

$-7.23956$

$-7.22598$

$-7.22331$

$L_{7.19943}$

$-7.16604$

$-7.14002$

47.08788

$-7.06706$

$-6.72693$

$-6.70024$

$L_{6.67350}$

$L_{6.64668}$

$L_{6.63044}$

$L_{6.60631}$

-6.59075

-6.58337

-6.58006

$-6.21390$

$-6.20267$

$L_{6.18785}$

$\leftarrow_{6.17656}$

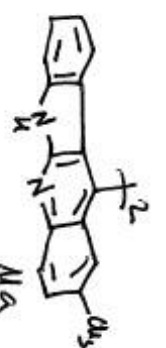

$+$

立

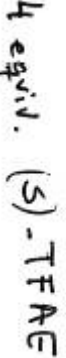

5
0
0
0
0
-1
7
7
7

5
0
0
$\vdots$
-1
7
7
7

5
0
0
0
-1
7
7
7

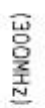




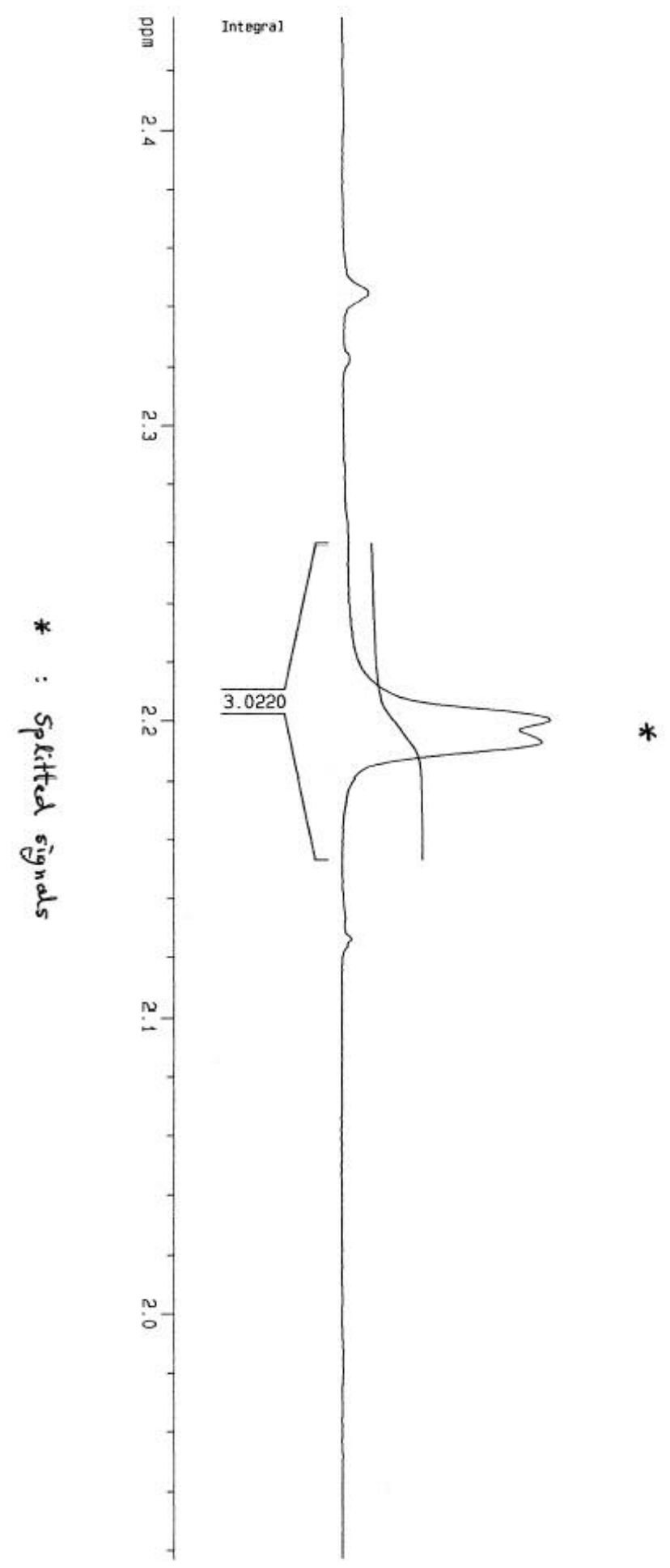

ppm

是

평

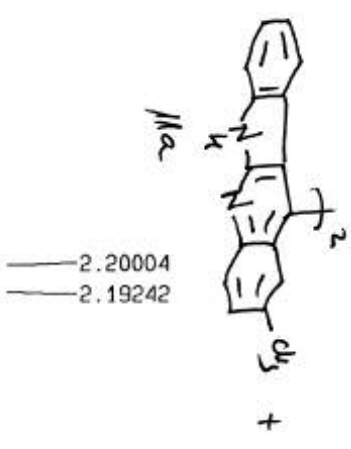

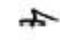

है

$\frac{\pi}{1}$

$+1$

花 

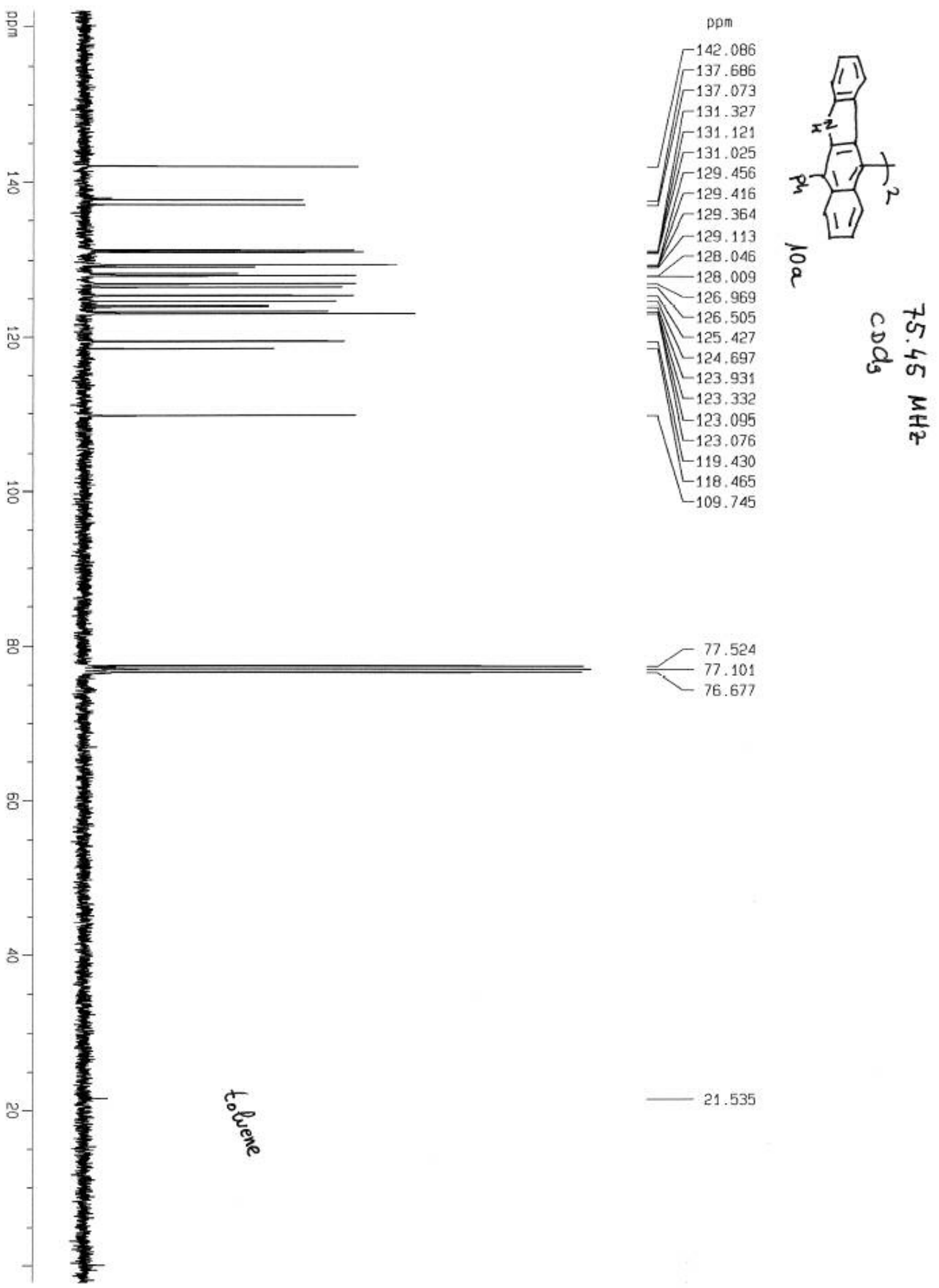

77.524

$-119.430$

$-109.745$

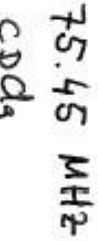

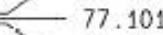

76.677
-701

21.535 

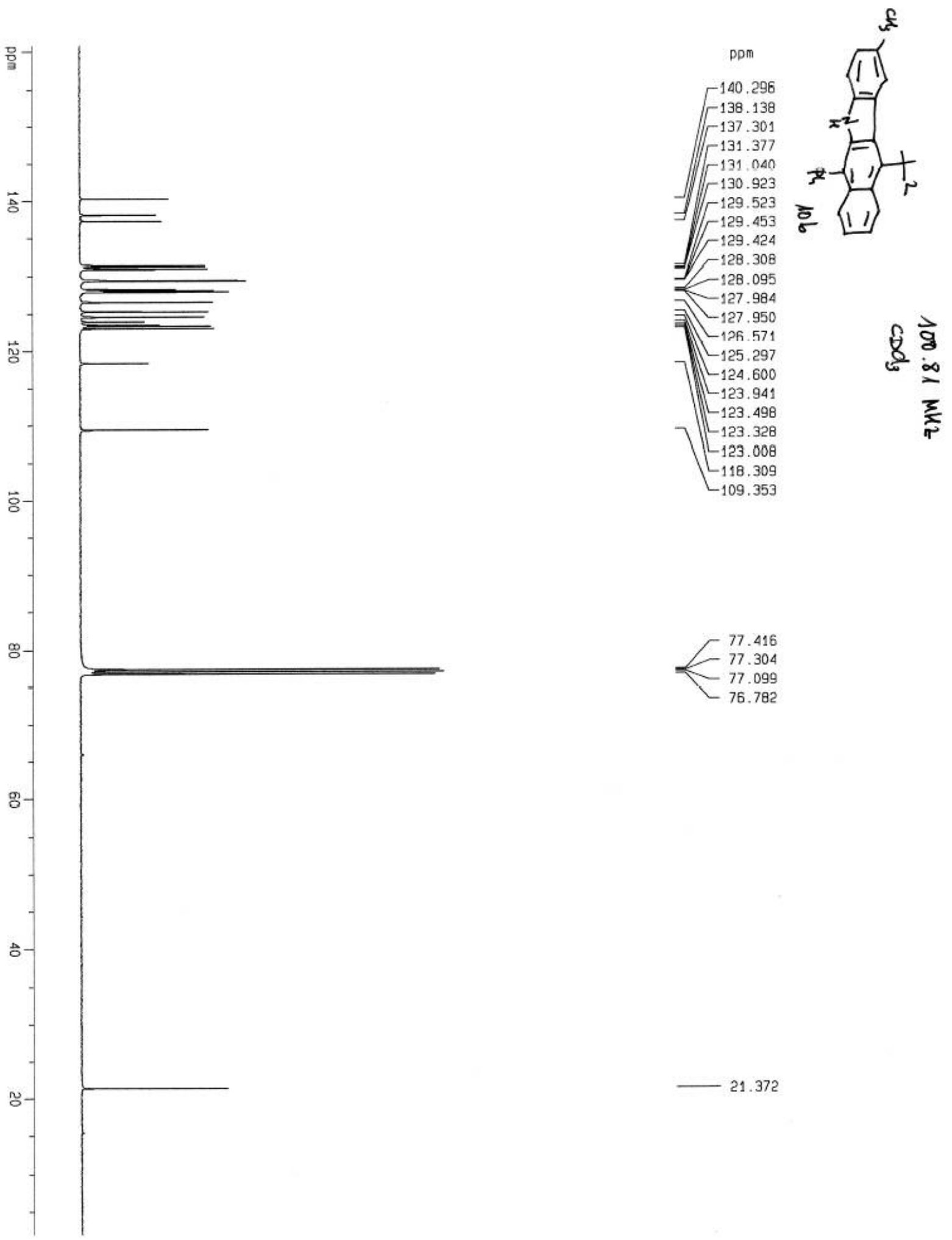

$-21.372$ 

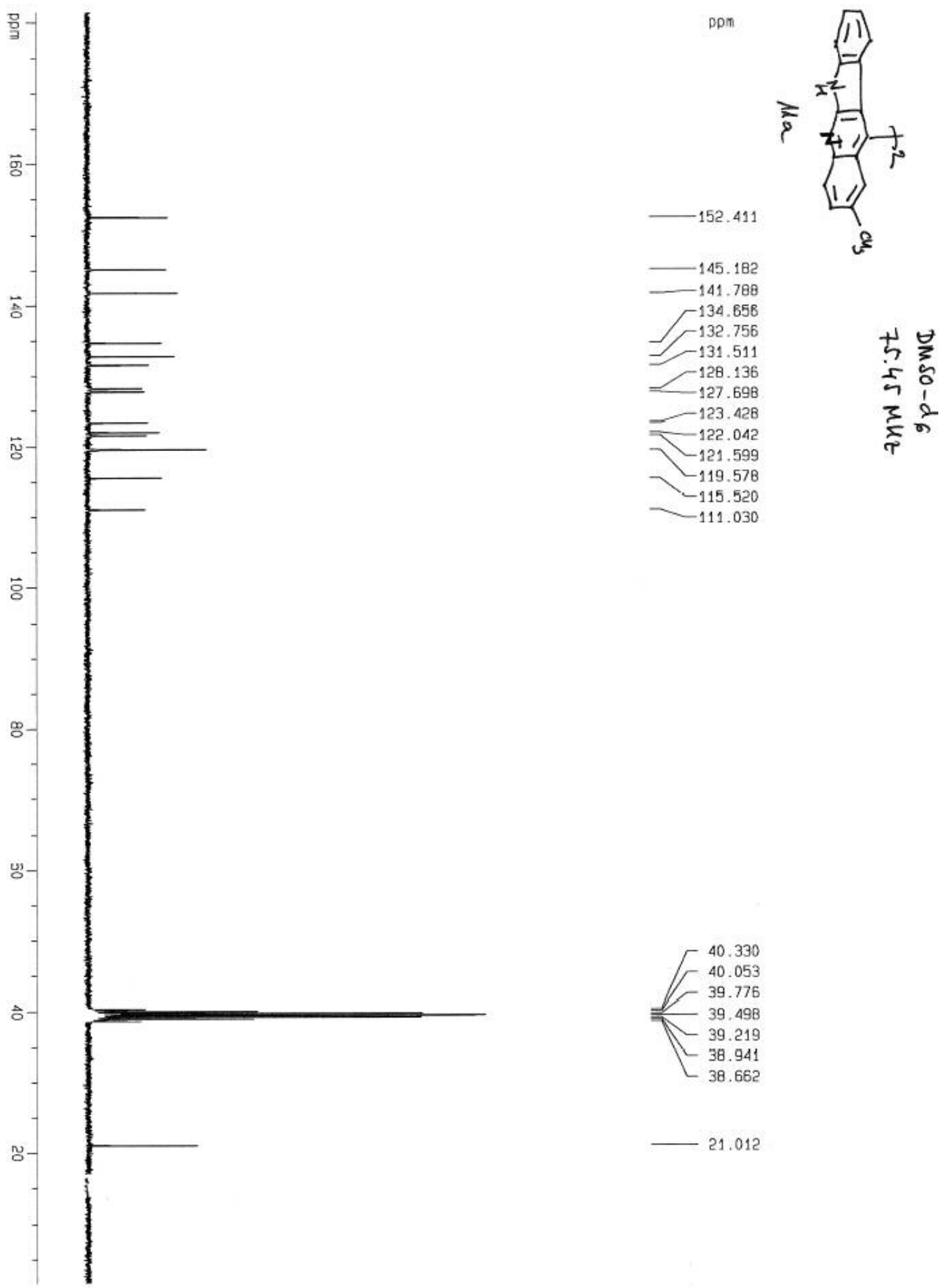

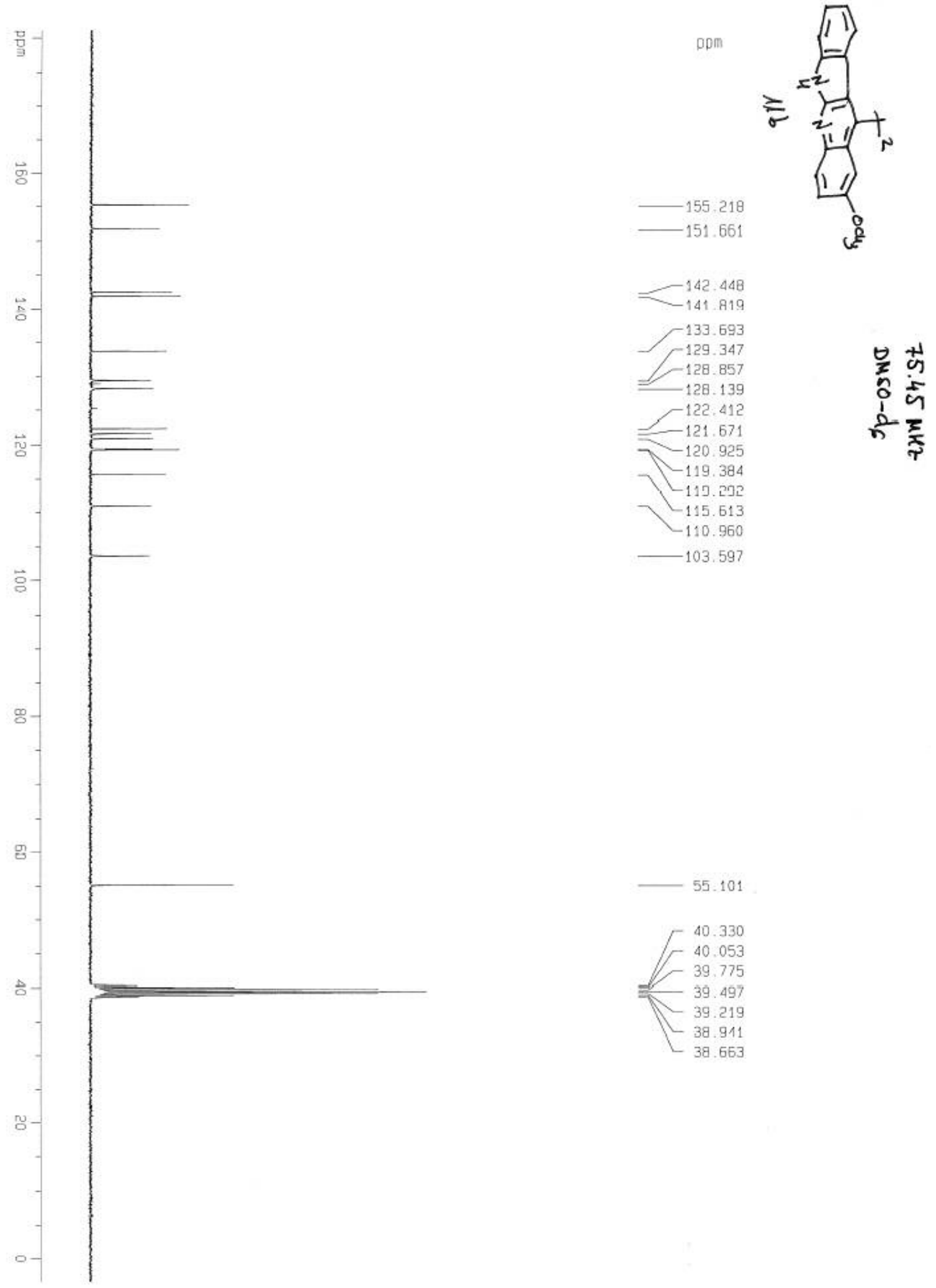


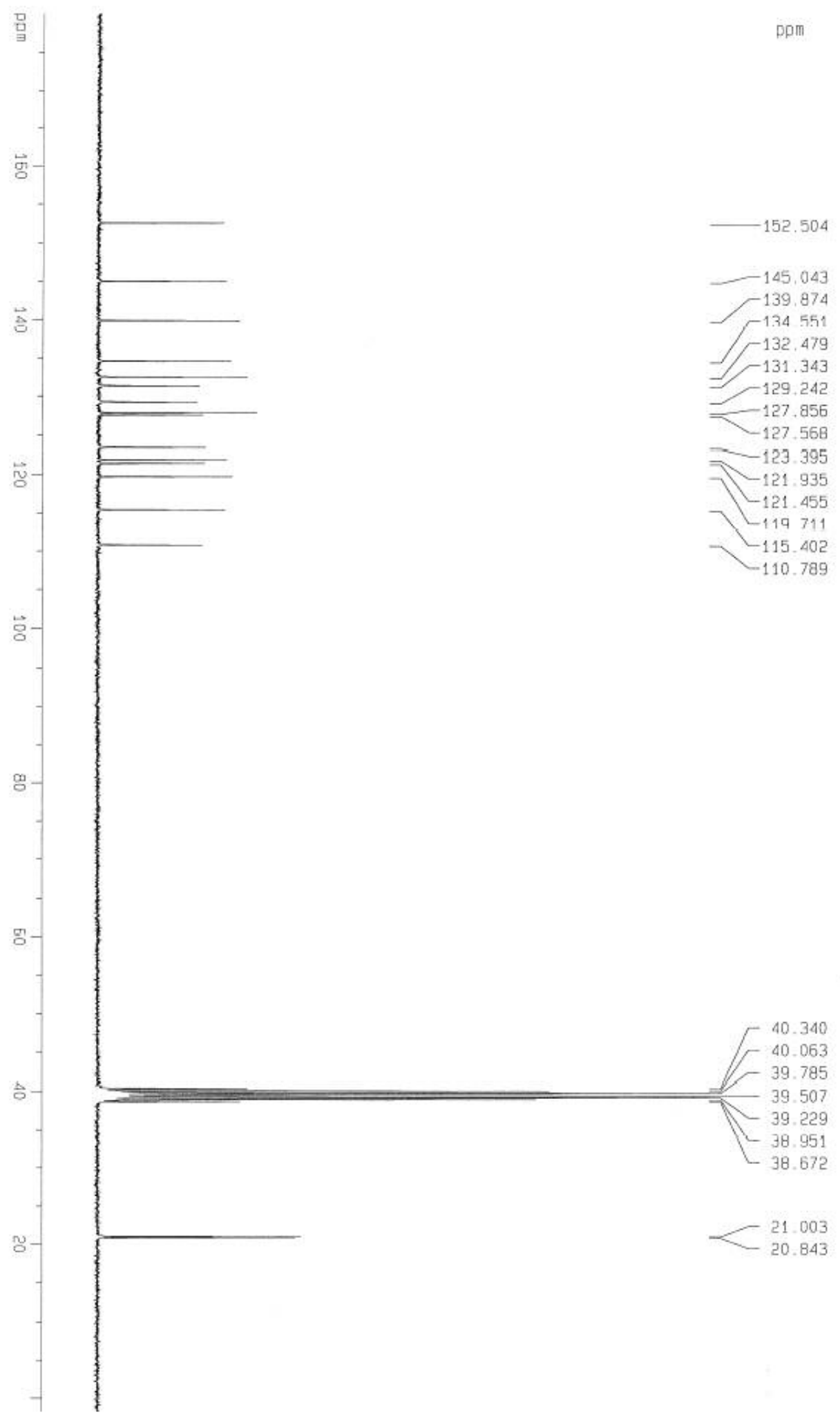



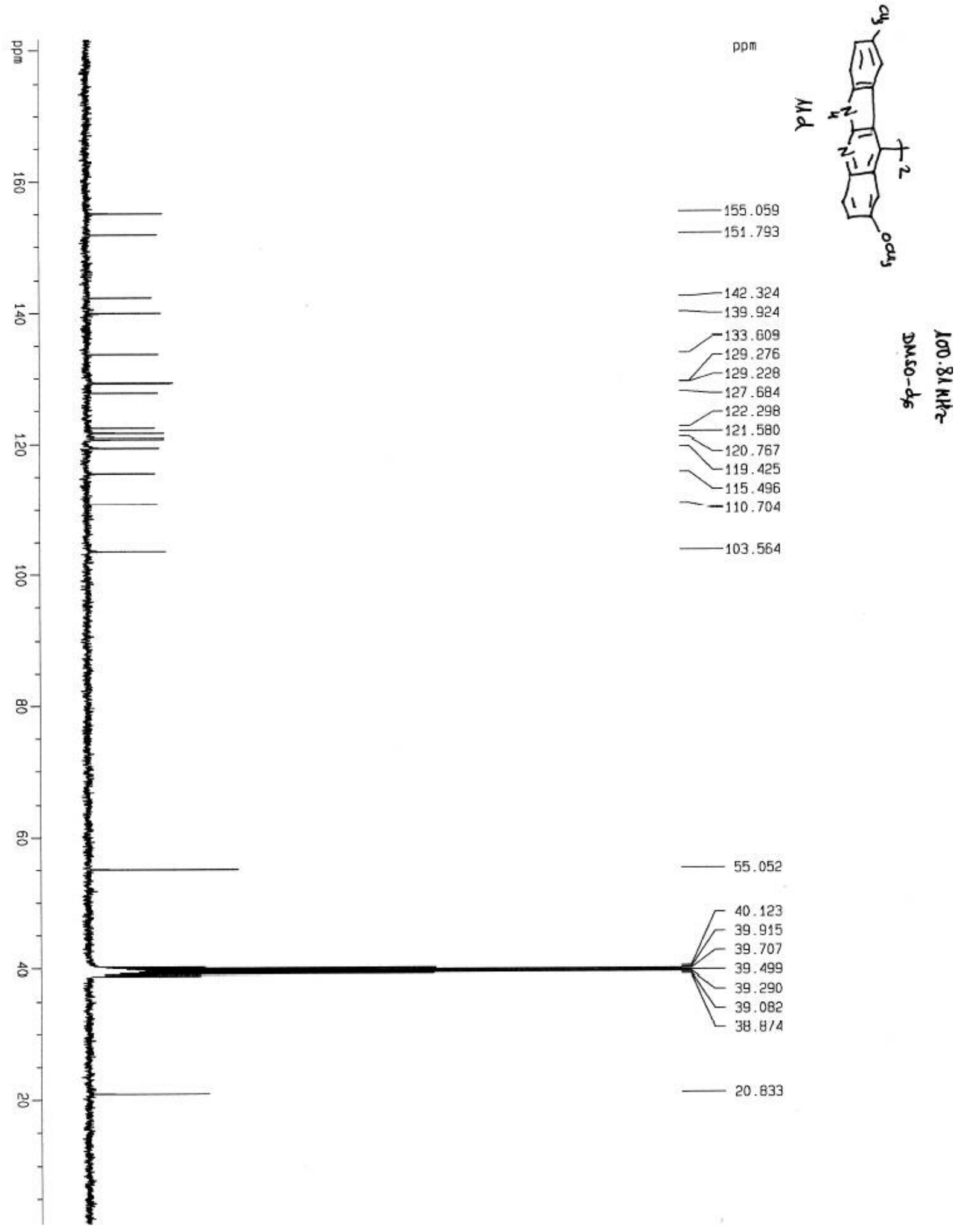

103.564

55.052

r 40.123

39.915

39.707

- 39.499

$-39.290$

- 39.082

$38.8 / 4$

20.833 

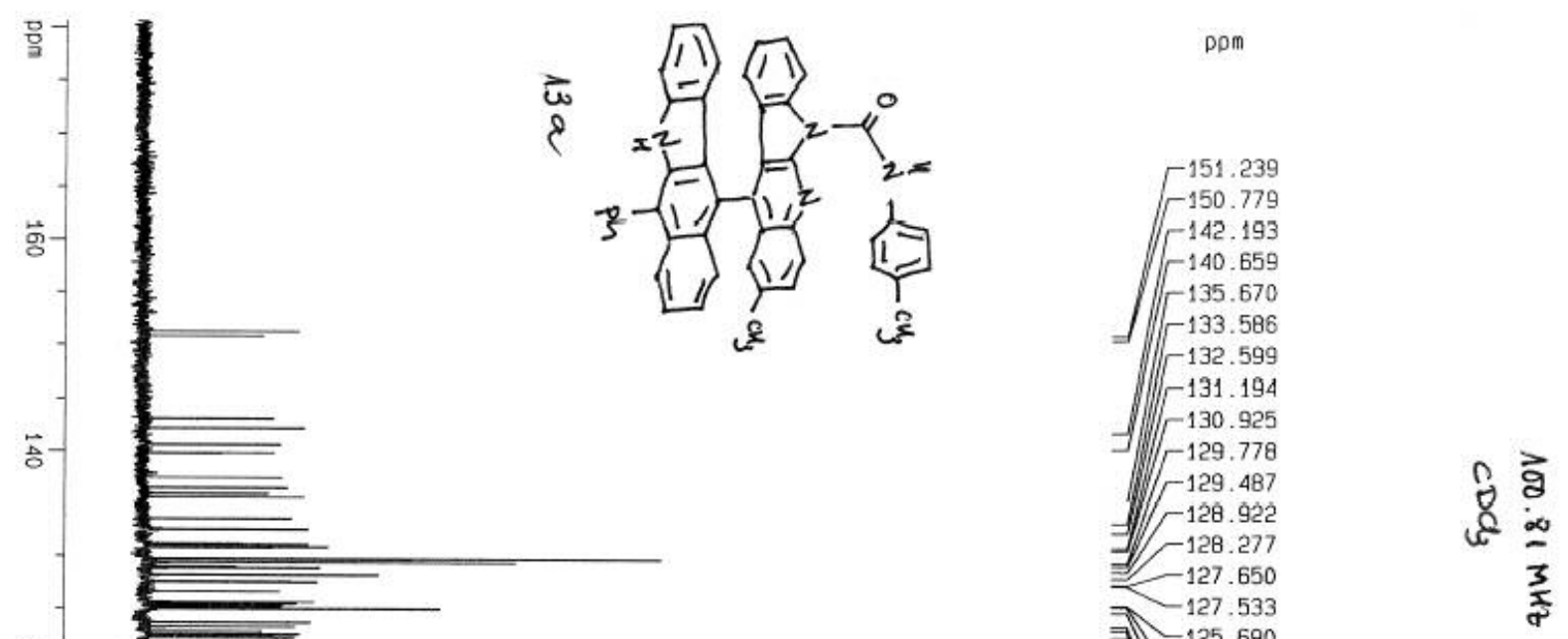

용

$-125.690$

$-125.528$

$L_{125.030}$

- -123.687

$\mathrm{L}_{1}-123.329$

(9.52. -128

$-122.301$

- 120.557

- 119.740

-116.798

$\leftarrow_{110.156}$

77.419

77.101
-76.785

䓢

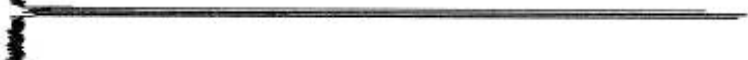

용

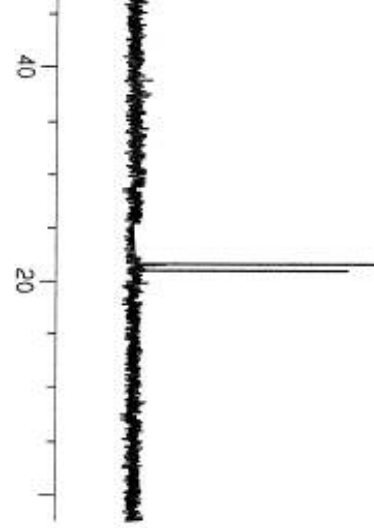

21.682

21.519

21.039 


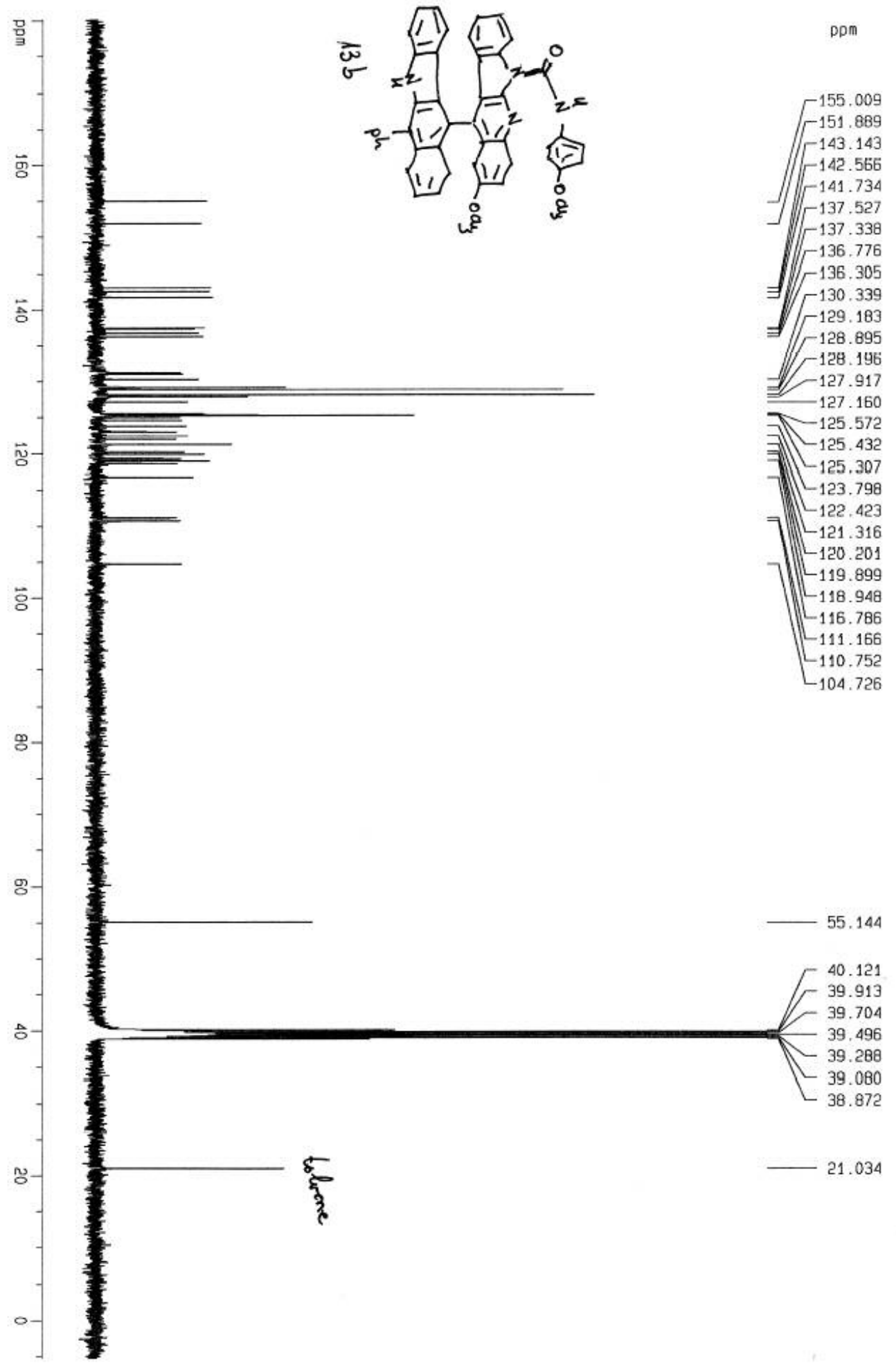




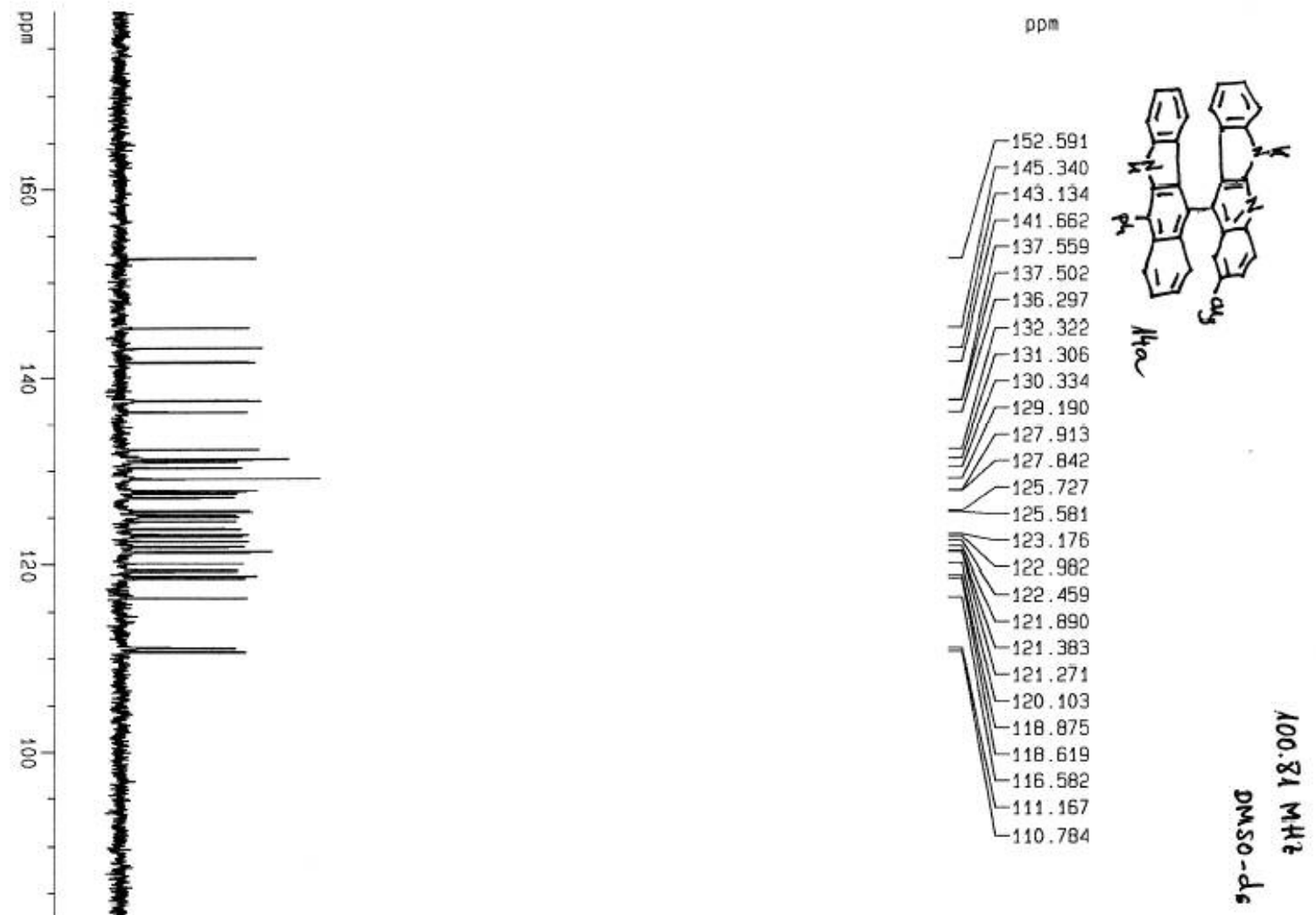

\%

oे

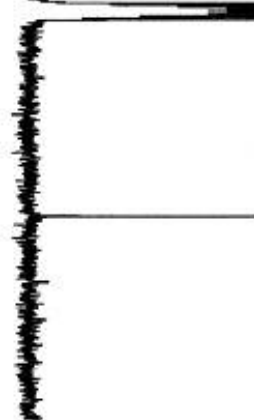

r 40.122

- 39.914

- 39.706

- 39.498

I 39.290

$-38.873$

ำ-

- 21.044 

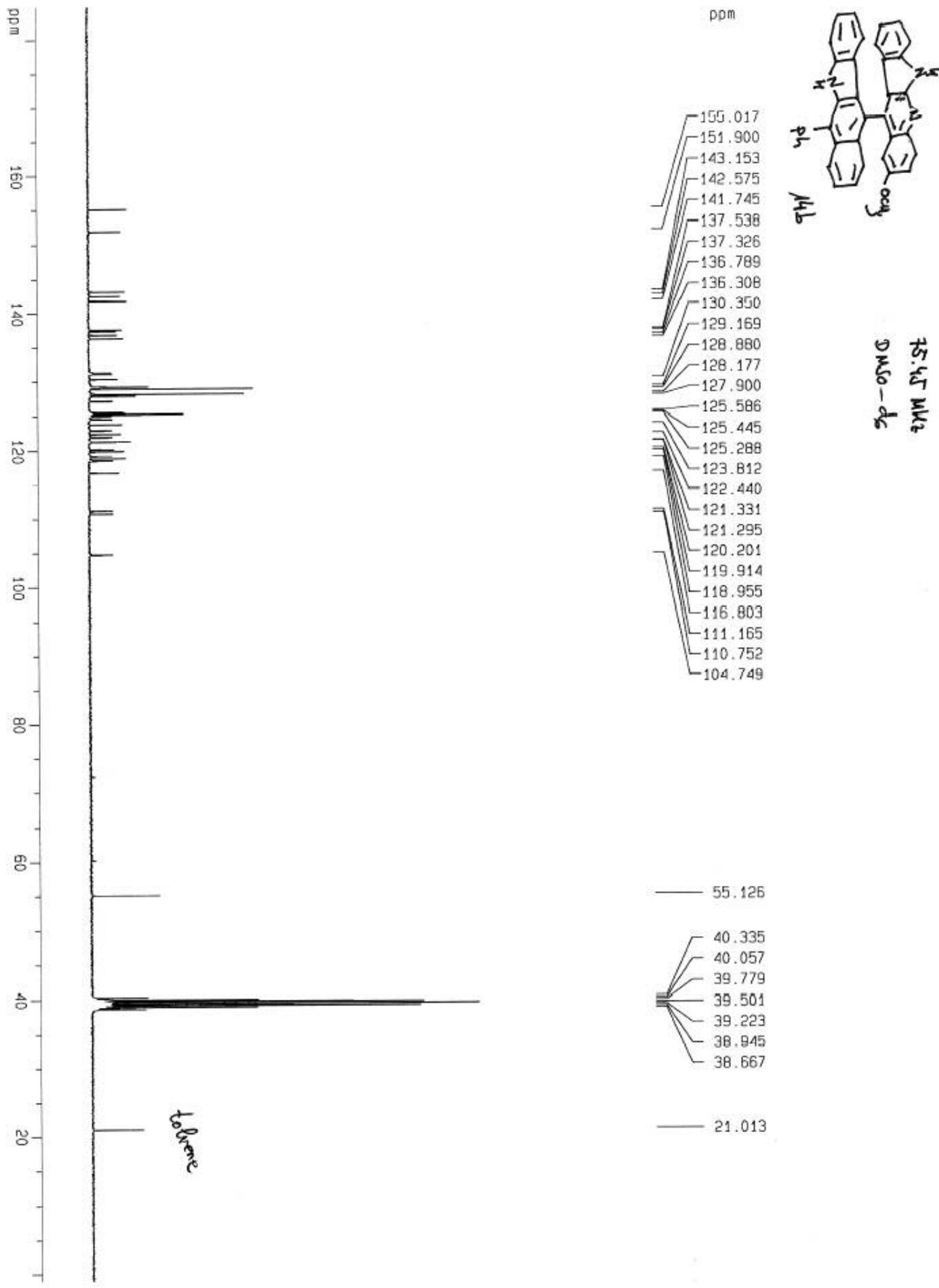

- 40.335

r 40.057

$-39.779$

39.501

— 39.223

$-38.945$

$-38.667$

21.013

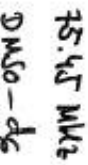

